Review

\title{
Longevity Extension by Phytochemicals
}

\author{
Anna Leonov ${ }^{\dagger}$, Anthony Arlia-Ciommo ${ }^{\dagger}$, Amanda Piano ${ }^{\dagger}$, Veronika Svistkova ${ }^{\dagger}$, \\ Vicky Lutchman ${ }^{\dagger}$, Younes Medkour ${ }^{\dagger}$ and Vladimir I. Titorenko * \\ Department of Biology, Concordia University, 7141 Sherbrooke Street, West, SP Building, \\ Room 501-13, Montreal, QC H4B 1R6, Canada; E-Mails: anna_leonova@yahoo.com (A.L.); \\ anthony.arlia@outlook.com (A.A.-C.); amandapiano91@hotmail.com (A.P.); \\ klubnika_veronika@hotmail.com (V.S.); lutchman.david@hotmail.com (V.L.); \\ writetoyounes@gmail.com (Y.M.) \\ $\dagger$ These authors contributed equally to this work. \\ * Author to whom correspondence should be addressed; E-Mail: vladimir.titorenko@concordia.ca; \\ Tel.: +1-514-848-2424 (ext. 3424); Fax: +1-514-848-2881.
}

Academic Editor: Marcello Iriti

Received: 2 March 2015 / Accepted: 8 April 2015 / Published: 13 April 2015

\begin{abstract}
Phytochemicals are structurally diverse secondary metabolites synthesized by plants and also by non-pathogenic endophytic microorganisms living within plants. Phytochemicals help plants to survive environmental stresses, protect plants from microbial infections and environmental pollutants, provide them with a defense from herbivorous organisms and attract natural predators of such organisms, as well as lure pollinators and other symbiotes of these plants. In addition, many phytochemicals can extend longevity in heterotrophic organisms across phyla via evolutionarily conserved mechanisms. In this review, we discuss such mechanisms. We outline how structurally diverse phytochemicals modulate a complex network of signaling pathways that orchestrate a distinct set of longevity-defining cellular processes. This review also reflects on how the release of phytochemicals by plants into a natural ecosystem may create selective forces that drive the evolution of longevity regulation mechanisms in heterotrophic organisms inhabiting this ecosystem. We outline the most important unanswered questions and directions for future research in this vibrant and rapidly evolving field.
\end{abstract}


Keywords: phytochemicals; interspecies chemical signals; aging; longevity regulation mechanisms; evolution; ecosystems; hormesis; signaling pathways; stress response; metabolism

\section{Introduction}

Plants use a diverse set of secondary biochemical pathways not to fulfill their primary metabolic needs in energy and biosynthetic products, but to generate a number of secondary metabolites called phytochemicals [1-5]. Some phytochemicals are produced not by plants, but by non-pathogenic endophytic bacteria and fungi that live within the plants [6-11].

Phytochemicals are structurally diverse chemical compounds; based on chemical nature, they can be divided into the following major classes: (1) phenolic compounds, including flavonoids, phenolic acids, hydroxycinnamic acids, lignans, tyrosol esters, stilbenoids and alkylresorcinols; (2) terpenes, including carotenoids, monoterpenes, saponins, some modified lipid species and triterpenoids; (3) betalains, including betacyanins and betaxanthins; (4) polysulfides; (5) organosulfur compounds; (6) indole compounds; (7) some protease inhibitors; (8) oxalic and anacardic organic acids; (9) modified purines; (10) quinones; and (11) polyamines [5,12-18]. It is believed that plants have evolved secondary biochemical pathways for synthesizing chemically diverse phytochemicals as ecosystemic adaptations; such evolutionary adaptations are thought to increase the chances of these immobile autotrophic organisms to survive and reproduce within their natural ecological niches [1-3,5,13,19-21]. Indeed, phytochemicals are used by plants as interspecies chemical signals that can: (1) help plants to survive various environmental stresses, including UV light, heat and cold stresses, osmotic stress and high salinity, extreme $\mathrm{pH}$, water deficit and dehydration, and nutrient deprivation; (2) protect plants from viral, bacterial, yeast and fungal infections; (3) defend plants from invading insects, herbivorous animals and competitor plant species; (4) provide plants with a protection from environmental pollutants; (5) attract pollinators and other symbiotes; and (6) attract the natural predators of herbivorous insects and animals [1,2,5,13,19-35]. Moreover, phytochemicals that are produced by non-pathogenic endophytic microorganisms living within the plants can promote the survival of the host plants by protecting them from being eaten by herbivorous insects and animals as well as by defending them from many environmental stresses and infections by pathogenic microorganisms [6-11,36-41].

A body of evidence supports the notion that, in addition to being beneficial to survival and reproduction of the plants producing them, phytochemicals can extend longevity and/or improve health in various heterotrophic organisms [5,13-18,21,22,27,39,42-60]. In this review, we discuss recent progress in understanding mechanisms underlying such longevity-extending and health-improving effects of phytochemicals on heterotrophic organisms across phyla. We also propose a hypothesis in which phytochemicals that have been released by plants into an ecosystem create xenohormetic, hormetic and cytostatic selective forces that may drive the evolution of longevity regulation mechanisms in heterotrophic organisms inhabiting this ecosystem. 


\section{Phytochemicals Extend Lifespan in Evolutionarily Distant Heterotrophic Organisms by Targeting an Evolutionarily Conserved Set of Longevity-Defining Cellular Processes}

Table 1 recapitulates numerous findings on how different phytochemicals prolong longevity in various heterotrophic organisms by modulating certain cellular processes [61-115]. The mechanisms by which these phytochemicals extend lifespan in organisms across phyla have begun to emerge. In this section, we discuss such mechanisms.

\subsection{Longevity-Extending Phytochemicals and Heterotrophic Organisms Whose Lifespans They Prolong}

Longevity-extending phytochemicals differ in chemical nature; they belong to various classes, including phenolic compounds [61,66,67,70,72,74-77,79,81-84,86,89,90-103,106-109,114,115], terpenes [73,78,85,87], polysulfides [80], organosulfur compounds [62-65], indole compounds [88,104,105], modified purines [68-71], quinones [17] and polyamines [111] (Table 1). These phytochemicals extend lifespan in such evolutionarily distant heterotrophic organisms and cultured cells as the budding yeast Saccharomyces cerevisiae [17,66,68,97,98,111], the fission yeast Schizosaccharomyces pombe [69], the nematode Caenorhabditis elegans (including a transgenic model of Alzheimer's disease) [67,70-72,74,80-87,89,90,99-101,103-106,111,114,115], the fruit fly Drosophila melanogaster (including different transgenic models of Alzheimer's disease) [61,75-77,94,106,111], the honey bee Apis mellifera [108], mosquitoes [95], the naturally short-lived fish Nothobranchius Furzeri [107], laboratory mice (including mice on a high-calorie diet and transgenic mice models of several age-related diseases) [62-65,73,78,91,92,109], laboratory rats [93], different lines of cultured human fibroblasts [79,88,96,102], and human peripheral blood mononuclear cells [111] (Table 1). It needs to be emphasized that some studies revealed that several of the longevity-extending phytochemicals mentioned in Table 1 are unable to prolong lifespan in certain heterotrophic organisms; for example, such phenolic compounds as resveratrol and curcumin did not alter the lifespan in genetically heterogeneous mice [116].

\subsection{Proteins and Signaling Pathways Required for Longevity Extension by Phytochemicals}

Cellular proteins and signaling pathways that are indispensable for lifespan-prolonging abilities of many longevity-extending phytochemicals have been identified (Table 1). They include the following proteins and pathways: (1) DAF-2, the only known receptor of the insulin/insulin-like growth factor 1 (IGF-1) signaling (IIS) pathway; this pathway defines longevity of the nematode C. elegans by regulating metabolism, protein homeostasis, resistance to many stresses, development and reproduction [70,71,89,101,115]; (2) the phosphatidylinositol 3-kinase AGE-1, an essential protein component of the IIS pathway in the nematode C. elegans [74,99-101]; (3) AKT-2, a serine/threonine protein kinase involved in the IIS pathway in the nematode $C$. elegans [72]; (4) SKN-1/Nrf, one of the transcription factors playing an essential role in the IIS pathway in the nematode C. elegans [74,80]; (5) the heat-shock factor 1 (HSF-1), a transcriptional factor involved in the IIS pathway in the nematode C. elegans [89,115]; (6) the transcription factor DAF-16/FOXO and its nuclear import in the nematode C. elegans - this protein is a key component of the IIS pathway $[67,70,71,81,86,89,90,99-101,114,115]$; (7) the OSR-1/UNC-43 (CaMKII)/SEK-1 (p38 MAPK) signaling pathway, which in the nematode 
C. elegans defines resistance to osmotic stress, arsenic, and pathogen infection $[67,74,101]$; (8) the nicotinic acetylcholine receptor EAT-2, which is essential for longevity regulation in the nematode C. elegans because it defines the rate of pharyngeal pumping in this organism [70,80,81,114]; (9) NHR-8, a non-canonical nuclear hormone receptor which is essential for longevity regulation-likely because it defines resistance to xenobiotic stress and plays essential roles in the metabolism of cholesterol, bile acids, and neutral lipids in the nematode C. elegans [72]; (10) the mitogen-activated protein kinase (MAPK) kinase MEK-1, which in the nematode $C$. elegans is involved in protein synthesis and stress-induced apoptosis and defines resistance to pathogen infection and heavy metals [74]; (11) the MEV-1 subunit of succinate-coenzyme Q oxidoreductase, a component of the mitochondrial electron transport chain that defines longevity of the nematode C. elegans [70,72,81,114]; (12) the histone acetyl transferase CBP-1, a transcriptional activator which is involved in mRNA processing and neurogenesis in the nematode $C$. elegans [70,71]; (13) TPH-1, a tryptophan hydroxlase enzyme involved in serotonin synthesis in the nematode C. elegans [104]; (14) the sirtuins Sir1 in the yeast S. cerevisiae [66], SIR-2.1 in the nematode C. elegans [67,74,106], Sir2 in the fruit fly D. melanogaster [106], and SIRT1 in mice on a high-calorie diet $[42,54,60,109]$ —all of which define longevity by modulating numerous cellular processes; (15) the target of rapamycin complex 1 (TORC1), which in the yeasts S. cerevisiae and Sch. pombe controls cell metabolism, protein synthesis, resistance to many stresses, and autophagy $[17,68,69]$; (16) the nutrient-sensing protein kinases Sch9 and Gcn2, which define longevity by modulating cell cycle progression, transcription, protein synthesis, responses to various stresses, amino acid synthesis and sphingolipid synthesis in the yeast $S$. cerevisiae [17]; (17) cytosolic and mitochondrial superoxide dismutases Sod1 and Sod2 (respectively), both playing essential roles in longevity regulation by detoxifying the superoxide radical, modulating cellular respiration, and controlling cell response to various stresses in the yeast $S$. cerevisiae [17,97]; and (18) the non-selective autophagy pathway for degradation of various cellular organelles and macromolecules in the yeast $S$. cerevisiae, nematode C. elegans, and fruit fly D. melanogaster [111-113].

\subsection{Processes Targeted by Longevity-Extending Phytochemicals in Evolutionarily Distant Organisms}

Longevity-extending phytochemicals have been shown to elicit changes in various cellular and organismal processes in organisms across phyla. These processes and organisms are outlined below and detailed in Table 1. 
Table 1. Phytochemicals that extend lifespan in various heterotrophic organisms and longevity-defining cellular processes that they modulate. Abbreviations: CaMK, $\mathrm{Ca}^{2+} /$ calmodulin-dependent protein kinase; FOXO, forkhead box protein O; HDTIC, 4-hydroxy-5-hydroxymethyl[1,3]dioxolan-2,6'-spirane-5',6',7', 8'-tetrahydro-indolizine-3'-carbaldehyde; Q3'G, quercetin 3'-O- $\beta$-D-glucopyranoside; Q3M, 3-O- $\beta$-Dglucopyranoside-(4 $\rightarrow 1)-\beta$-D-glucopyranoside; MAPK, mitogen-activated protein kinase; NT, not tested; rDNA, ribosomal DNA; ROS, reactive oxygen species.

\begin{tabular}{|c|c|c|c|c|c|}
\hline Phytochemical & Plant & Chemical Nature & $\begin{array}{c}\text { Organism } \\
\text { Exhibiting Lifespan } \\
\text { Extension } \\
\end{array}$ & $\begin{array}{c}\text { Cellular Proteins and } \\
\text { Signaling Pathways } \\
\text { Required } \\
\end{array}$ & Changes Caused \\
\hline Acteoside & $\begin{array}{l}\text { Phlomis anisodonta, Phlomis bruguieri, } \\
\text { Verbascum phlomoides, Verbascum } \\
\text { mallophorum, Buddleja globose, } \\
\text { Buddleja cordata }\end{array}$ & $\begin{array}{l}\text { Caffeoyl phenylethanoid } \\
\text { glycoside (a phenolic } \\
\text { compound) }\end{array}$ & $\begin{array}{l}\text { The fruit fly } \\
\text { Drosophila } \\
\text { melanogaster [61] }\end{array}$ & NT & NT \\
\hline Allicin & Allium sativum (garlic) & Organosulfur compound & $\begin{array}{l}- \text { Senescence- } \\
\text { accelerated } \\
\text { mice }[62-65]\end{array}$ & NT & $\begin{array}{l}\text { - Improved memory retention } \\
\text { and acquisition [62-65] }\end{array}$ \\
\hline Butein & Toxicodendron vernicifluum & $\begin{array}{l}\text { Chalconoid (a phenolic } \\
\text { compound) }\end{array}$ & $\begin{array}{l}\text { - The yeast } \\
\text { Saccharomyces } \\
\text { cerevisiae [66] }\end{array}$ & - The sirtuin Sir1 [66] & NT \\
\hline $\begin{array}{l}\text { Caffeic acid, } \\
\text { rosmarinic acid }\end{array}$ & Eucalyptus globulus, Salvinia molesta & $\begin{array}{l}\text { Hydroxycinnamic acids } \\
\text { (phenolic compounds) }\end{array}$ & $\begin{array}{l}\text { - The nematode } \\
\text { Caenorhabditis } \\
\text { elegans [67] }\end{array}$ & $\begin{array}{l}\text { - The OSR-1/UNC-43 } \\
(\mathrm{CaMKII}) / \mathrm{SEK}-1 \text { (p38 } \\
\text { MAPK) signaling pathway } \\
{[67]} \\
\text { - The sirtuin SIR-2.1 [67] } \\
\text { - Caffeic acid only: the } \\
\text { DAF-16/FOXO transcription } \\
\text { factor [67] }\end{array}$ & $\begin{array}{l}\text { - Lowered susceptibility to } \\
\text { thermal stress [67] } \\
\text { - Decreased oxidative damage } \\
\text { to macromolecules [67] } \\
\text { - Reduced body size, altered } \\
\text { lipid metabolism, delayed } \\
\text { reproductive timing [67] }\end{array}$ \\
\hline
\end{tabular}


Table 1. Cont.

\begin{tabular}{|c|c|c|c|c|c|}
\hline Phytochemical & Plant & Chemical Nature & $\begin{array}{l}\text { Organism Exhibiting } \\
\text { Lifespan Extension }\end{array}$ & $\begin{array}{c}\text { Cellular Proteins and Signaling Pathways } \\
\text { Required }\end{array}$ & Changes Caused \\
\hline Caffeine & $\begin{array}{l}\text { Coffea } \\
\text { plants }\end{array}$ & $\begin{array}{l}\text { Methylxanthine (a } \\
\text { purine) }\end{array}$ & $\begin{array}{l}\text { - The yeasts Saccharomyces } \\
\text { cerevisiae [68] and } \\
\text { Schizosaccharomyces pombe } \\
\text { [69] } \\
\text { - The nematode C. elegans } \\
\text { [70,71] }\end{array}$ & $\begin{array}{l}\text { - In } S . \text { cerevisiae and Sch. pombe: the target } \\
\text { of rapamycin complex } 1 \text { (TORC1) }[68,69] \\
\text { - In C. elegans: the insulin-like receptor } \\
\text { DAF-2, transcription factor DAF-16/FOXO } \\
\text { and transcriptional activator CBP-1 [70,71] }\end{array}$ & $\begin{array}{l}\text { - In S. cerevisiae: enhanced transcription of } \\
\text { genes encoding heat-shock proteins and } \\
\text { molecular chaperones [68] } \\
\text { - In Sch. pombe: decelerated growth, G2 } \\
\text { cell-cycle arrest, altered transcription of } \\
\text { many nuclear genes, attenuated protein } \\
\text { synthesis and inhibited phosphorylation of } \\
\text { ribosomal S6 proteins [69] } \\
\text { - In C. elegans: delayed onset of paralysis } \\
\text { and reduced protein aggregation in } \\
\text { nematode models of the Alzheimer's and } \\
\text { Huntington's diseases [70,71] }\end{array}$ \\
\hline Catechin & $\begin{array}{l}\text { Vascular } \\
\text { plants }\end{array}$ & $\begin{array}{l}\text { Flavan-3-ol (a } \\
\text { phenolic } \\
\text { compound) }\end{array}$ & - The nematode C. elegans [72] & $\begin{array}{l}\text { - The AKT-2 serine/threonine protein kinase, } \\
\text { MEV-1 subunit of succinate-coenzyme Q } \\
\text { oxidoreductase in the mitochondrial } \\
\text { electron transport chain, and nuclear } \\
\text { hormone receptor NHR- } 8 \text { [72] }\end{array}$ & $\begin{array}{l}\text { - Reduced body length and susceptibility to } \\
\text { thermal stress [72] } \\
\text { - Elevated pumping rate [72] }\end{array}$ \\
\hline Celastrol & $\begin{array}{l}\text { Tripterygium } \\
\text { wilfordii, } \\
\text { Celastrus } \\
\text { regelii }\end{array}$ & $\begin{array}{l}\text { Triterpenoid (a } \\
\text { terpen) }\end{array}$ & $\begin{array}{l}\text { - Transgenic mouse model of } \\
\text { amyotrophic lateral sclerosis } \\
\text { (ALS) [73] }\end{array}$ & NT & $\begin{array}{l}\text { - Decelerated weight loss, improved motor } \\
\text { performance, increased number of neurons } \\
\text { and delayed onset of ALS [73] }\end{array}$ \\
\hline
\end{tabular}


Table 1. Cont.

\begin{tabular}{|c|c|c|c|c|c|}
\hline Phytochemical & Plant & Chemical Nature & $\begin{array}{l}\text { Organism Exhibiting } \\
\text { Lifespan Extension }\end{array}$ & $\begin{array}{c}\text { Cellular Proteins and Signaling } \\
\text { Pathways Required }\end{array}$ & Changes Caused \\
\hline $\begin{array}{l}\text { Curcumin, } \\
\text { tetrahydrocurcumin }\end{array}$ & Curcuma longa & $\begin{array}{l}\text { Diarylheptanoids } \\
\text { (phenolic } \\
\text { compounds) }\end{array}$ & $\begin{array}{l}\text { - The nematode } \\
\text { C. elegans [74] } \\
\text { - The fruit fly } \\
\text { D. melanogaster, } \\
\text { including } 5 \text { different } \\
\text { models of Alzheimer's } \\
\text { disease [75-77] }\end{array}$ & $\begin{array}{l}\text { - In C. elegans: the OSR-1/UNC- } \\
43 \text { (CaMKII)/SEK-1 (p38 MAPK) } \\
\text { signaling pathway [74] } \\
\text { - In C. elegans: the sirtuin SIR-2.1 } \\
\text { [74] } \\
\text { - In C. elegans: the } \\
\text { phosphatidylinositol 3-kinase } \\
\text { AGE-1, transcription factor SKN- } \\
\text { 1/Nrf and MAPK kinase } \\
\text { MEK-1 [74] }\end{array}$ & $\begin{array}{l}\text { - In C. elegans: Reduced ROS levels, } \\
\text { macromolecular oxidative damage, } \\
\text { susceptibility to oxidative and } \\
\text { thermal stresses, body length, and } \\
\text { pumping rate [74] } \\
\text { - In D. melanogaster: Decreased } \\
\text { macromolecular oxidative damage, } \\
\text { lowered susceptibility to oxidative } \\
\text { stress, improved locomotor } \\
\text { performance [75-77] }\end{array}$ \\
\hline Crocin & Crocus, Gardenia & $\begin{array}{l}\text { Carotenoid (a } \\
\text { terpen) }\end{array}$ & $\begin{array}{l}\text { - Dalton's lymphoma } \\
\text { ascites bearing mice } \\
{[78]}\end{array}$ & NT & $\begin{array}{l}\text { - Increased hemoglobin and } \\
\text { lymphocytes [78] } \\
\text { - Decreased white blood cell count } \\
\text { and neutrophils [78] }\end{array}$ \\
\hline Cryptotanshinone & Salvia miltiorrhiza & $\begin{array}{l}\text { Tanshion (a } \\
\text { quinone) }\end{array}$ & $\begin{array}{l}\text { - The yeast } S \text {. } \\
\text { cerevisiae [17] }\end{array}$ & $\begin{array}{l}\text { - Mitochondrial superoxide } \\
\text { dismutase Sod2, as well as the } \\
\text { nutrient-sensing protein kinases } \\
\text { Tor1, Sch9 and Gcn2 [17] }\end{array}$ & - Lowered ROS levels [17] \\
\hline Cyanidin & $\begin{array}{l}\text { Vitis vinifera, Vitis labrusca, } \\
\text { Vaccinium myrtillus, } \\
\text { Vaccinium uliginosum, } \\
\text { Vaccinium alaskaense, } \\
\text { Vaccinium angustifolium } \\
\end{array}$ & $\begin{array}{l}\text { Anthocyanidin (a } \\
\text { phenolic compound) }\end{array}$ & $\begin{array}{l}\text { - WI-38 human diploid } \\
\text { fibroblasts [79] }\end{array}$ & NT & $\begin{array}{l}\text { - Reduced oxidative damage to lipids } \\
\text { and susceptibility to oxidative stress } \\
\text { [79] }\end{array}$ \\
\hline
\end{tabular}


Table 1. Cont.

\begin{tabular}{|c|c|c|c|c|c|}
\hline Phytochemical & Plant & $\begin{array}{l}\text { Chemical } \\
\text { Nature }\end{array}$ & $\begin{array}{c}\text { Organism } \\
\text { Exhibiting } \\
\text { Lifespan } \\
\text { Extension } \\
\end{array}$ & $\begin{array}{c}\text { Cellular Proteins and } \\
\text { Signaling Pathways } \\
\text { Required }\end{array}$ & Changes Caused \\
\hline Diallyl trisulfide & Allium sativum (garlic) & $\begin{array}{l}\text { A polysulfide (an } \\
\text { organosulfide } \\
\text { compound) }\end{array}$ & $\begin{array}{l}- \text { The nematode } \\
\text { C. elegans }[80]\end{array}$ & $\begin{array}{l}\text { - The nicotinic } \\
\text { acetylcholine receptor } \\
\text { EAT-2 and } \\
\text { transcription factor } \\
\text { SKN-1/Nrf [80] }\end{array}$ & $\begin{array}{l}\text { - Altered expression of many nuclear genes involved in } \\
\text { metabolism and stress response [80] }\end{array}$ \\
\hline Ellagic acid & $\begin{array}{l}\text { Quercus alba, Quercus } \\
\text { robur, Myriophyllum } \\
\text { spicatum }\end{array}$ & $\begin{array}{l}\text { Phenolic acid (a } \\
\text { phenolic } \\
\text { compound) }\end{array}$ & $\begin{array}{l}- \text { The nematode } \\
\text { C. elegans [81] }\end{array}$ & $\begin{array}{l}\text { - The nicotinic } \\
\text { acetylcholine receptor } \\
\text { EAT-2 [81] }\end{array}$ & $\begin{array}{l}\text { - Delayed beginning of egg deposition and reduced oxidative } \\
\text { damage to water-soluble metabolites [81] }\end{array}$ \\
\hline $\begin{array}{l}\text { Epigallocatechin } \\
\text { gallate }\end{array}$ & Camellia sinensis & $\begin{array}{l}\text { Flavan-3-ol (a } \\
\text { phenolic } \\
\text { compound) }\end{array}$ & $\begin{array}{l}\text { - The nematode } \\
\text { C. elegans } \\
{[82,83]}\end{array}$ & NT & $\begin{array}{l}\text { - Lowered ROS levels, reduced susceptibility to oxidative stress, } \\
\text { decreased oxidative damage to lipids, attenuated expression of } \\
\text { nuclear genes encoding HSP-16, induced nuclear import of the } \\
\text { transcription factor DAF-16/FOXO, reduced formation of A } \beta \\
\text { deposits }[82,83]\end{array}$ \\
\hline Epicatechin & $\begin{array}{l}\text { Seed of Theobroma cacao, } \\
\text { juice of Prunus domestica, } \\
\text { seed of Vicia faba, oil } \\
\text { from the fruit of Euterpe } \\
\text { oleracea }\end{array}$ & $\begin{array}{l}\text { Flavan-3-ol (a } \\
\text { phenolic } \\
\text { compound) }\end{array}$ & $\begin{array}{l}\text { - The fruit fly } \\
\text { Drosophila } \\
\text { melanogaster } \\
\text { [84] } \\
\text { - Obese diabetic } \\
\text { mice [84] }\end{array}$ & NT & $\begin{array}{l}\text { - In obese diabetic mice: reduced degeneration of aortic vessels, } \\
\text { lowered fat deposition, decreased hydropic degeneration in the } \\
\text { liver, reduced markers of systematic inflammation, lowered } \\
\text { serum LDL cholesterol, decreased level of circulating insulin- } \\
\text { like growth factor 1, improved skeletal muscle stress output, } \\
\text { increased concentration of hepatic glutathione, elevated } \\
\text { superoxide dismutase activity, amplified AMP-activated protein } \\
\text { kinase activity in the liver and skeletal muscle [84] }\end{array}$ \\
\hline
\end{tabular}


Table 1. Cont.

\begin{tabular}{|c|c|c|c|c|c|}
\hline Phytochemical & Plant & Chemical Nature & $\begin{array}{c}\text { Organism } \\
\text { Exhibiting } \\
\text { Lifespan } \\
\text { Extension } \\
\end{array}$ & $\begin{array}{l}\text { Cellular Proteins and } \\
\text { Signaling Pathways } \\
\text { Required }\end{array}$ & Changes Caused \\
\hline Ferulsinaic acid & Ferula plants & $\begin{array}{l}\text { Sesquiterpene } \\
\text { coumarin (a } \\
\text { terpene) }\end{array}$ & $\begin{array}{l}\text { - The nematode } \\
\text { C. elegans }[85]\end{array}$ & NT & $\begin{array}{l}\text { - Reduced susceptibility to oxidative and thermal } \\
\text { stresses, decreased oxidative damage to lipids, lowered } \\
\text { formation of advanced glycation end products [85] }\end{array}$ \\
\hline Fisetin & $\begin{array}{l}\text { Acacia greggii, Acacia } \\
\text { berlandieri, Butea frondosa, } \\
\text { Gleditsia triacanthos, } \\
\text { Quebracho colorado, Rhus } \\
\text { cotinus }\end{array}$ & $\begin{array}{l}\text { Flavonol (a } \\
\text { phenolic } \\
\text { compound) }\end{array}$ & $\begin{array}{l}\text { - The yeast } S \text {. } \\
\text { cerevisiae }[66] \\
\text { - The nematode } \\
\text { C. elegans }[86]\end{array}$ & $\begin{array}{l}\text { - In S. cerevisiae: The sirtuin } \\
\text { Sir1 [66] } \\
\text { - In C. elegans: Nuclear } \\
\text { import of the transcription } \\
\text { factor DAF-16/FOXO [86] }\end{array}$ & $\begin{array}{l}\text { - In S. cerevisiae: NT [66] } \\
\text { - In C. elegans: Lowered ROS levels, reduced } \\
\text { susceptibility to oxidative stress, decreased oxidative } \\
\text { damage to macromolecules, induced nuclear import of } \\
\text { transcription factor DAF-16/FOXO [86] }\end{array}$ \\
\hline Gallic acid & $\begin{array}{l}\text { Quercus alba, Quercus robur, } \\
\text { Caesalpinia mimosoides, } \\
\text { Boswellia dalzielii, Rhodiola } \\
\text { rosea, } \\
\text { Toona sinensis }\end{array}$ & $\begin{array}{l}\text { Phenolic acid (a } \\
\text { phenolic } \\
\text { compound) }\end{array}$ & $\begin{array}{l}\text { - The nematode } \\
\text { C. elegans }[81]\end{array}$ & $\begin{array}{l}\text { - The nicotinic acetylcholine } \\
\text { receptor EAT-2 [81] }\end{array}$ & $\begin{array}{l}\text { - Increased body length, delayed beginning of egg } \\
\text { deposition and reduced oxidative damage to water- } \\
\text { soluble metabolites [81] }\end{array}$ \\
\hline Glaucarubinone & Simaroubaceae plants & $\begin{array}{l}\text { Triterpenoid (a } \\
\text { terpen) }\end{array}$ & $\begin{array}{l}\text { - The nematode } \\
\text { C. elegans }[87]\end{array}$ & NT & $\begin{array}{l}\text { - Increased rate of oxygen consumption and lowered } \\
\text { levels of neutral lipids [87] }\end{array}$ \\
\hline $\begin{array}{l}\text { HDTIC-1, } \\
\text { HDTIC-2 }\end{array}$ & Astragalus membranceus & $\begin{array}{l}\text { Indolizines (indole } \\
\text { compounds) }\end{array}$ & $\begin{array}{l}\text { - Human fetal } \\
\text { lung diploid } \\
\text { fibroblasts [88] }\end{array}$ & NT & $\begin{array}{l}\text { - Improved growth and proliferation, accelerated entry } \\
\text { from G0 or G1 phase to S phase, decreased activity of } \\
\text { the senescence-associated- } \beta \text {-galactosidase, and } \\
\text { reduced formation of advanced glycation end products } \\
\text { [88] }\end{array}$ \\
\hline $\begin{array}{l}\text { Icariin, icariside } \\
\text { II }\end{array}$ & Epimedium plants & $\begin{array}{l}\text { Flavonol } \\
\text { glycosides } \\
\text { (phenolic } \\
\text { compounds) }\end{array}$ & $\begin{array}{l}\text { - The nematode } \\
\text { C. elegans [89] }\end{array}$ & $\begin{array}{l}\text { The insulin-like receptor } \\
\text { DAF-2, transcription factor } \\
\text { DAF-16/FOXO and heat } \\
\text { shock transcription factor } \\
\text { HSF-1 [89] }\end{array}$ & $\begin{array}{l}\text { - Reduced susceptibility to oxidative and thermal } \\
\text { stresses, decelerated decline in age-related locomotion, } \\
\text { delayed onset of paralysis caused by the proteotoxicity } \\
\text { of polyQ and A } \beta(1-42) \text {, enhanced transcription of the } \\
\text { SOD-3 and HSP-12.3 genes [89] }\end{array}$ \\
\hline
\end{tabular}


Table 1. Cont.

\begin{tabular}{|c|c|c|c|c|c|}
\hline Phytochemical & Plant & Chemical Nature & $\begin{array}{l}\text { Organism } \\
\text { Exhibiting } \\
\text { Lifespan } \\
\text { Extension }\end{array}$ & $\begin{array}{l}\text { Cellular Proteins and } \\
\text { Signaling Pathways } \\
\text { Required }\end{array}$ & Changes Caused \\
\hline Kaempferol & $\begin{array}{l}\text { Aloe vera, Coccinia grandis, } \\
\text { Cuscuta chinensis, Euphorbia } \\
\text { pekinensis, Glycine max, } \\
\text { Hypericum perforatum }\end{array}$ & $\begin{array}{l}\text { Flavonol (a } \\
\text { phenolic } \\
\text { compound) }\end{array}$ & $\begin{array}{l}\text { - The nematode } C \text {. } \\
\text { elegans }[86]\end{array}$ & $\begin{array}{l}\text { - Nuclear import of } \\
\text { the transcription factor } \\
\text { DAF-16/FOXO [86] }\end{array}$ & $\begin{array}{l}\text { - Lowered ROS levels, reduced susceptibility to } \\
\text { oxidative stress, decreased oxidative damage to } \\
\text { macromolecules, induced nuclear import of } \\
\text { transcription factor DAF-16/FOXO [86] }\end{array}$ \\
\hline Myricetin & $\begin{array}{l}\text { Morella rubra, Myrica } \\
\text { cerifera, Rosa damascene, } \\
\text { Salvia hispanica, } \\
\text { Hovenia dulcis, Ceratonia } \\
\text { siliqua }\end{array}$ & $\begin{array}{l}\text { Flavonol (a } \\
\text { phenolic } \\
\text { compound) }\end{array}$ & $\begin{array}{l}\text { - The nematode } C \text {. } \\
\text { elegans }[90]\end{array}$ & $\begin{array}{l}\text { - Nuclear import of } \\
\text { the transcription factor } \\
\text { DAF-16/FOXO [90] }\end{array}$ & $\begin{array}{l}\text { - Lowered ROS levels, reduced oxidative damage to } \\
\text { proteins, induced nuclear import of transcription } \\
\text { factor DAF-16/FOXO, enhanced transcription of the } \\
\text { SOD-3 gene [90] }\end{array}$ \\
\hline $\begin{array}{l}\text { Nordihydroguaiaretic } \\
\text { acid }\end{array}$ & Larrea tridentata & $\begin{array}{l}\text { Lignan (a phenolic } \\
\text { compound) }\end{array}$ & $\begin{array}{l}\text { - Transgenic } \\
\text { mouse model of } \\
\text { ALS [91] } \\
\text { - Male mice [92] } \\
\text { - Rats [93] } \\
\text { - The fruit fly } D . \\
\text { melanogaster [94] } \\
\text { - Mosquitoes [95] }\end{array}$ & NT & $\begin{array}{l}\text { - In transgenic mouse model of ALS: reduced motor } \\
\text { dysfunction [91] } \\
\text { - In D. melanogaster: lowered rate of oxygen } \\
\text { consumption [94] }\end{array}$ \\
\hline Oleuropein & Olea europaea & $\begin{array}{l}\text { Phenylethanoid (a } \\
\text { phenolic } \\
\text { compound) }\end{array}$ & $\begin{array}{l}\cdot \text { Human } \\
\text { embryonic } \\
\text { fibroblasts [96] }\end{array}$ & NT & $\begin{array}{l}\text { - Lowered ROS levels, reduced oxidative damage to } \\
\text { proteins, increased rate of proteasomal degradation } \\
\text { of oxidatively damaged proteins, decelerated age- } \\
\text { related decline in proteasome activity [96] }\end{array}$ \\
\hline
\end{tabular}


Table 1. Cont.

\begin{tabular}{|c|c|c|c|c|c|}
\hline Phytochemical & Plant & $\begin{array}{c}\text { Chemical } \\
\text { Nature }\end{array}$ & $\begin{array}{c}\text { Organism Exhibiting } \\
\text { Lifespan Extension }\end{array}$ & $\begin{array}{c}\text { Cellular Proteins and Signaling } \\
\text { Pathways Required }\end{array}$ & Changes Caused \\
\hline Phloridzin & $\begin{array}{l}\text { Pyrus communis, } \\
\text { Malus domestica, } \\
\text { Prunus avium, } \\
\text { Rosaceae plants, } \\
\text { Dianthus caryophyllus }\end{array}$ & $\begin{array}{l}\text { Chalconoid } \\
\text { (a phenolic } \\
\text { compound) }\end{array}$ & $\begin{array}{l}\text { - The yeast } S \text {. cerevisiae } \\
\text { [97] }\end{array}$ & $\begin{array}{l}\text { - Cytosolic and mitochondrial } \\
\text { superoxide dismutases Sod1 and Sod2 } \\
\text { (respectively) [97] }\end{array}$ & $\begin{array}{l}\text { - Lowered ROS levels, decreased susceptibility } \\
\text { to oxidative stress, activated transcription of the } \\
\text { SOD1, SOD2 and SIR } 2 \text { genes, increased } \\
\text { superoxide dismutase activity [97] }\end{array}$ \\
\hline $\begin{array}{l}\text { Quercetin, } \\
\text { Q3'G, Q3M, } \\
\text { isorhamnetin, } \\
\text { tamarixetin }\end{array}$ & $\begin{array}{l}\text { Capparis spinosa, } \\
\text { Levisticum officinale, } \\
\text { Rumex acetosa, } \\
\text { Raphanus sativus, } \\
\text { Ceratonia siliqua, } \\
\text { Anethum graveolens }\end{array}$ & $\begin{array}{l}\text { Flavonols } \\
\text { (phenolic } \\
\text { compounds) }\end{array}$ & $\begin{array}{l}\text { - The yeast } S \text {. cerevisiae } \\
\text { [98] } \\
\text { - The nematode } C \text {. } \\
\text { elegans }[67,99-101,103] \\
\text { - Human embryonic } \\
\text { fibroblasts [102] }\end{array}$ & $\begin{array}{l}\text { - In C. elegans: the insulin-like receptor } \\
\text { DAF-2, phosphatidylinositol 3-kinase } \\
\text { AGE-1 and nuclear import of the } \\
\text { transcription factor DAF-16/FOXO } \\
\text { [99-101], as well as the OSR-1/UNC- } \\
43 \text { (CaMKII)/SEK-1 (p38 MAPK) } \\
\text { signaling pathway [101] }\end{array}$ & $\begin{array}{l}\text { - In S. cerevisiae: Lowered ROS levels, } \\
\text { decreased glutathione oxidation, reduced protein } \\
\text { carbonylation, lowered lipid peroxidation, } \\
\text { decreased susceptibility to oxidative stress [98] } \\
\text { - In C. elegans: Lowered ROS levels, reduced } \\
\text { oxidative damage to macromolecules, enhanced } \\
\text { anti-oxidative activities, decreased susceptibility } \\
\text { to thermal and oxidative stresses, lowered level } \\
\text { of neutral lipids, induced nuclear import of } \\
\text { transcription factor DAF-16/FOXO [67,99-101] } \\
\text { - In human fibroblasts: Lowered activity of the } \\
\text { senescence-associated- } \beta \text {-galactosidase, } \\
\text { decreased ROS levels, reduced susceptibility to } \\
\text { oxidative stress, increased proteasome activity } \\
\text { [102] }\end{array}$ \\
\hline
\end{tabular}


Table 1. Cont.

\begin{tabular}{|c|c|c|c|c|c|}
\hline Phytochemical & Plant & $\begin{array}{c}\text { Chemical } \\
\text { Nature }\end{array}$ & $\begin{array}{c}\text { Organism Exhibiting } \\
\text { Lifespan Extension }\end{array}$ & $\begin{array}{c}\text { Cellular Proteins and } \\
\text { Signaling Pathways } \\
\text { Required }\end{array}$ & Changes Caused \\
\hline Reserpine & Rauvolfia serpentina & $\begin{array}{l}\text { Indole alkaloid } \\
\text { (an indole } \\
\text { compound) }\end{array}$ & $\begin{array}{l}\text { - The nematode } C \text {. } \\
\text { elegans }[104] \\
\text { - The nematode } C \text {. } \\
\text { elegans model of } \\
\text { Alzheimer's disease } \\
{[105]}\end{array}$ & $\begin{array}{l}- \text { TPH-1, a tryptophan } \\
\text { hydroxlase enzyme } \\
{[104] .}\end{array}$ & $\begin{array}{l}\text { - Reduced susceptibility to thermal stress, decelerated decline in } \\
\text { age-related locomotion and pharyngeal pumping, delayed } \\
\text { postembryonic development }[104,105] \\
\text { - The nematode } C \text {. elegans model of Alzheimer's disease: } \\
\text { delayed onset of paralysis caused by the proteotoxicity of A } \beta \\
{[105]}\end{array}$ \\
\hline Resveratrol & $\begin{array}{l}\text { Vitis plants, Vaccinium } \\
\text { alaskaense, } \\
\text { Vaccinium angustifolium, } \\
\text { Rubus idaeus, Rubus } \\
\text { occidentalis, } \\
\text { Broussonetia papyrifera }\end{array}$ & $\begin{array}{l}\text { Stilbenoid (a } \\
\text { phenolic } \\
\text { compound) }\end{array}$ & $\begin{array}{l}\text { - The yeast } S \text {. } \\
\text { cerevisiae }[66] \\
\text { - The nematode } C \text {. } \\
\text { elegans }[106] \\
\text { - The fruit fly D. } \\
\text { melanogaster [106] } \\
\text { - The short-lived fish } \\
\text { Nothobranchius } \\
\text { Furzeri }[107] \\
\text { - The honey bee Apis } \\
\text { mellifera }[108] \\
\text { - Mice on a high- } \\
\text { calorie diet [109] }\end{array}$ & $\begin{array}{l}\text { - In S. cerevisiae: the } \\
\text { sirtuin Sir1 [66] } \\
\text { - The nematode } C \text {. } \\
\text { elegans: the sirtuin } \\
\text { SIR-2.1 [106] } \\
\text { - The fruit fly D. } \\
\text { melanogaster: the } \\
\text { sirtuin Sir2 [106] } \\
\text { - Mouse: SIRT1 and } \\
\text { many other cellular } \\
\text { targets, direct or } \\
\text { indirect }[42,54,60]\end{array}$ & $\begin{array}{l}\text { - In } S \text {. cerevisiae: reduced frequency of rDNA recombination } \\
\text { [66] } \\
\text { - In the nematode C. elegans: induced autophagy [110] } \\
\text { - In } N \text {. furzeri: delayed age-related decay of locomotor activity } \\
\text { and cognitive performances, reduced neurofibrillary } \\
\text { degeneration in the brain [107] } \\
\text { - In mouse: increased insulin sensitivity, increased activities of } \\
\text { AMP-activated protein kinase (AMPK) and peroxisome } \\
\text { proliferator-activated receptor-gamma coactivator } 1 \alpha \text { (PGC-1 } \alpha \text { ), } \\
\text { reduced levels of insulin-like growth factor-1 (IGF-I), increased } \\
\text { number of mitochondria, altered transcription of many nuclear } \\
\text { genes [109] }\end{array}$ \\
\hline
\end{tabular}


Table 1. Cont.

\begin{tabular}{|c|c|c|c|c|c|}
\hline Phytochemical & Plant & $\begin{array}{l}\text { Chemical } \\
\text { Nature }\end{array}$ & $\begin{array}{l}\text { Organism } \\
\text { Exhibiting } \\
\text { Lifespan } \\
\text { Extension } \\
\end{array}$ & $\begin{array}{c}\text { Cellular Proteins and Signaling } \\
\text { Pathways Required }\end{array}$ & Changes Caused \\
\hline $\begin{array}{l}\text { Spermidine, } \\
\text { putrescine }\end{array}$ & $\begin{array}{l}\text { Wheat (Triticum spp.) } \\
\text { germ, Ipomoea } \\
\text { batatas, Pisum } \\
\text { sativum, Glycine max, } \\
\text { Glycine soja }\end{array}$ & Polyamines & $\begin{array}{l}\text { - The yeast } S \text {. } \\
\text { cerevisiae }[111] \\
\text { - The nematode } C \text {. } \\
\text { elegans }[111] \\
\text { - The fruit fly } D . \\
\text { melanogaster } \\
\text { [111] } \\
\text { - Human } \\
\text { peripheral blood } \\
\text { mononuclear cells } \\
\text { (PBMC) [111] }\end{array}$ & $\begin{array}{l}- \text { In } S . \text { cerevisiae, C. elegans and } D . \\
\text { melanogaster: autophagy }[111,112]\end{array}$ & $\begin{array}{l}\text { - In } S \text {. cerevisiae, D. melanogaster and PBMC: } \\
\text { lowered activities of histone acetyltransferases, } \\
\text { increased histone H3 deacetylation, activated } \\
\text { transcription of many autophagy-related genes, } \\
\text { induced autophagy, delayed onset of age-related } \\
\text { necrotic cell death, reduced age-related decline of } \\
\text { locomotor activity [111] } \\
\text { - In } D \text {. melanogaster: } \\
\text { decelerated age-related decline of locomotor } \\
\text { activity, increased level of triglycerides, altered } \\
\text { relative levels of fatty acid species and } \\
\text { phospholipid classes [112,113] }\end{array}$ \\
\hline Tannic acid & $\begin{array}{l}\text { Caesalpinia spinosa, } \\
\text { Rhus semialata, } \\
\text { Quercus infectoria, } \\
\text { Rhus coriaria }\end{array}$ & $\begin{array}{l}\text { Polyphenol (a } \\
\text { phenolic } \\
\text { compound) }\end{array}$ & $\begin{array}{l}\text { - The nematode } C \text {. } \\
\text { elegans } \\
{[70,81,114]}\end{array}$ & $\begin{array}{l}\text { - The mitogen-activated protein kinase } \\
\text { kinase SEK-1, transcription factor DAF- } \\
\text { 16/FOXO, nicotinic acetylcholine } \\
\text { receptor EAT-2 and MEV-1 subunit of } \\
\text { succinate-coenzyme Q oxidoreductase in } \\
\text { the mitochondrial electron transport } \\
\text { chain }[70,81,114]\end{array}$ & $\begin{array}{l}\text { - Reduced body length, decreased susceptibility to } \\
\text { thermal and oxidative stresses, lowered levels of } \\
\text { triglycerides, enhanced anti-oxidant capacity } \\
{[70,81,114]}\end{array}$ \\
\hline Tyrosol & $\begin{array}{l}\text { Oil from the fruit of } \\
\text { Olea europaea, oil } \\
\text { from the kernels of } \\
\text { Argania spinosa, } \\
\text { leaves from Camellia } \\
\text { sinensis }\end{array}$ & $\begin{array}{l}\text { Phenylethanoid (a } \\
\text { phenolic } \\
\text { compound) }\end{array}$ & $\begin{array}{l}\text { - The nematode } C \text {. } \\
\text { elegans }[115]\end{array}$ & $\begin{array}{l}\text { - The insulin-like receptor DAF-2, } \\
\text { transcription factor DAF-16/FOXO and } \\
\text { heat shock transcription factor HSF-1 } \\
{[115]}\end{array}$ & $\begin{array}{l}\text { - Decreased susceptibility to thermal and oxidative } \\
\text { stresses, decelerated onset of age-related decline in } \\
\text { pharyngeal pumping, activated transcription of } \\
\text { nuclear genes encoding several heat-shock proteins } \\
\text { [115] }\end{array}$ \\
\hline
\end{tabular}




\subsubsection{Yeasts}

In the yeast $S$. cerevisiae, the changes elicited by longevity-extending phytochemicals include the following: (1) caffeine enhances transcription of genes encoding heat-shock proteins and molecular chaperones [68]; (2) cryptotanshinone reduces cellular levels of reactive oxygen species (ROS) [17]; (3) phloridzin decreases cellular levels of ROS, increases resistance to oxidative stress and superoxide dismutase activity, and activates transcription of the SOD1 (cytosolic superoxide dismutase), SOD2 (mitochondrial superoxide dismutase) and SIR2 (sirtuin) genes [97]; (4) quercetin reduces cellular levels of ROS, the efficiencies of glutathione oxidation and lipid peroxidation, the extent of protein carbonylation, and cell susceptibility to oxidative stress [98]; (5) resveratrol decreases the frequency of rDNA recombination [66]; and (6) spermidine reduces activities of histone acetyltransferases, increases the extent of histone $\mathrm{H} 3$ deacetylation, activates transcription of many autophagy-related genes, induces autophagy and delays onset of age-related necrotic cell death [111] (Table 1). In the yeast Sch. pombe, caffeine decelerates growth, causes cell-cycle arrest in G2, alters transcription of many nuclear genes, attenuates protein synthesis and inhibits phosphorylation of ribosomal S6 proteins [69] (Table 1).

\subsubsection{The Nematode C. elegans}

In the nematode $C$. elegans, longevity-extending phytochemicals cause the following changes: (1) caffeic and rosmarinic acids decrease susceptibility to thermal stress, reduce oxidative damage to macromolecules, lower body size, alter lipid metabolism and delay reproductive timing [67]; (2) caffeine delays the onset of paralysis and reduces protein aggregation in nematode models of Alzheimer's and Huntington's diseases [70,71]; (3) catechin lowers body length, reduces susceptibility to thermal stress, and elevates pumping rate [72]; (4) curcumin and tetrahydrocurcumin decrease cellular levels of ROS, the extent of oxidative damage to macromolecules, susceptibility to oxidative and thermal stresses, body length, and pumping rate [74]; (5) diallyl trisulfide alters expression of many nuclear genes involved in metabolism and stress response [80]; (6) ellagic acid delays the beginning of egg deposition and lowers the extent of oxidative damage to water-soluble metabolites [81]; (7) epigallocatechin gallate lowers cellular levels of ROS, reduces susceptibility to oxidative stress, decreases the extent of oxidative damage to lipids, attenuates expression of nuclear genes encoding HSP-16, enhances nuclear import of the transcription factor DAF-16/FOXO, and mitigates the formation of A $\beta$ deposits [82,83]; (8) ferulsinaic acid reduces susceptibility to oxidative and thermal stresses, lowers the extent of oxidative damage to lipids, and slows down the formation of advanced glycation end products [85]; (9) fisetin decreases cellular levels of ROS, lowers susceptibility to oxidative stress, reduces the extent of oxidative damage to macromolecules and stimulates nuclear import of the transcription factor DAF-16/FOXO [86]; (10) gallic acid increases body length, delays the beginning of egg deposition, and reduces the extent of oxidative damage to water-soluble metabolites [81]; (11) glaucarubinone increases the rate of oxygen consumption and reduces cellular levels of neutral lipids [87]; (12) icariin and icariside II lower susceptibility to oxidative and thermal stresses, decelerate age-related decline in locomotion, delay the onset of paralysis elicited by the proteotoxicity of polyQ and $\mathrm{A} \beta(1-42)$, and stimulate transcription of the $S O D-3$ and HSP-12.3 genes [89]; (13) kaempferol lowers cellular levels of ROS, reduces susceptibility to oxidative stress, decreases the extent of oxidative damage to macromolecules, and accelerates nuclear import of 
the transcription factor DAF-16/FOXO [86]; (14) myricetin decreases cellular levels of ROS, lowers the extent of oxidative damage to proteins, stimulates nuclear import of the transcription factor DAF-16/FOXO and enhances transcription of the SOD-3 gene [90]; (15) quercetin lowers cellular levels of ROS, decreases the extent of oxidative damage to macromolecules, elevates anti-oxidative activities, reduces susceptibility to thermal and oxidative stresses, reduces cellular levels of neutral lipids, and stimulates nuclear import of the transcription factor DAF-16/FOXO [67,99-101]; (16) reserpine decreases susceptibility to thermal stress, decelerates the age-related declines in locomotion and pharyngeal pumping, and delays postembryonic development [104,105]; in the nematode model of Alzheimer's disease it also postpones the onset of paralysis caused by the proteotoxicity of $A \beta[105]$; (17) resveratrol and spermidine induce autophagy [110,111]; (18) tannic acid decreases body length, lowers susceptibility to thermal and oxidative stresses, reduces cellular levels of triglycerides, and enhances anti-oxidant capacity [70,81,114]; and (19) tyrosol lowers susceptibility to thermal and oxidative stresses, decelerates the onset of an age-related decline in pharyngeal pumping, and stimulates transcription of nuclear genes encoding several heat-shock proteins [115] (Table 1).

\subsubsection{The Fruit Fly D. melanogaster}

In the fruit fly $D$. melanogaster, the alterations caused by longevity-extending phytochemicals include the following: (1) curcumin and tetrahydrocurcumin lower the extent of macromolecular oxidative damage, reduce susceptibility to oxidative stress, and improve locomotor performance [75-77]; (2) nordihydroguaiaretic acid decreases the rate of oxygen consumption [94]; and (3) spermidine lowers susceptibility to oxidative stress, induces autophagy, decelerates age-related decline of locomotor activity, increases cellular levels of triglycerides, and alters relative levels of fatty acid species and phospholipid classes [112,113] (Table 1).

\subsubsection{The Fish Nothobranchius Furzeri}

In the naturally short-lived fish $N$. furzeri, resveratrol delays age-related decay of locomotor activity and cognitive performances [107]. This phenolic phytochemical is also known to reduce neurofibrillary degeneration in the brain of $N$. furzeri [107] (Table 1).

\subsubsection{Laboratory Mouse}

In laboratory mice (including transgenic mice models of several age-related diseases and mice on a high-calorie diet), longevity-extending phytochemicals elicit the following changes: (1) allicin improves memory retention and acquisition in senescence-accelerated mice models [62-65]; (2) celastrol decelerates weight loss, improves motor performance, increases the number of neurons and delays the onset of amyotrophic lateral sclerosis (ALS) in a transgenic mouse model of ALS [73]; (3) crocin increases hemoglobin and lymphocytes, and decreases white blood cell count and neutrophils in Dalton's lymphoma ascites-bearing mice [78]; (4) epicatechin reduces degeneration of aortic vessels and fat deposition, decreases hydropic degeneration in the liver and markers of systematic inflammation, lowers levels of serum LDL cholesterol and circulating insulin-like growth factor 1, improves skeletal muscle stress output, increases concentration of hepatic glutathione and total superoxide dismutase activity, and 
elevates AMP-activated protein kinase activity in diabetic mice [84]; (5) nordihydroguaiaretic acid reduces motor dysfunction in a transgenic mouse model of ALS [91]; and (6) resveratrol increases insulin sensitivity, stimulates activities of AMP-activated protein kinase (AMPK) and peroxisome proliferator-activated receptor-gamma coactivator $1 \alpha(\mathrm{PGC}-1 \alpha)$, lowers levels of insulin-like growth factor-1 (IGF-I), elevates the number of mitochondria, and alters transcription of many nuclear genes in mice on a high-calorie diet [109] (Table 1).

\subsubsection{Cultured Human Cells}

In cultured human cells, the alterations caused by longevity-extending phytochemicals include the following: (1) cyanidin lowers oxidative damage to lipids and decreases susceptibility to oxidative stress in WI-38 human diploid fibroblasts [79]; (2) two 4-hydroxy-5-hydroxymethyl-[1,3]dioxolan-2,6'spirane-5',6',7', 8'-tetrahydro-indolizine-3'-carbaldehydes (HDTIC), HDTIC-1, and HDTIC-2 improve growth and proliferation, accelerate entry from $\mathrm{G} 0$ or $\mathrm{G} 1$ phase to $\mathrm{S}$ phase of the cell cycle, lower activity of the senescence-associated- $\beta$-galactosidase, and decrease formation of advanced glycation end products in human fetal lung diploid fibroblasts [88]; (3) oleuropein lowers cellular levels of ROS, reduces oxidative damage to proteins, increases the rate of proteasomal degradation of oxidatively damaged proteins, and decelerates age-related decline in proteasome activity in human embryonic fibroblasts [96]; (4) quercetin lowers the activity of the senescence-associated- $\beta$-galactosidase, decreases cellular levels of ROS, reduces susceptibility to oxidative stress, and stimulates proteasome activity in human embryonic fibroblasts [102]; and (5) spermidine lowers the extent of histone $\mathrm{H} 3$ acetylation in human peripheral blood mononuclear cells (PBMC) and induces autophagy in human HeLa cells [111] (Table 1).

\subsection{Mechanisms of Longevity Extension by Phytochemicals Are Evolutionarily Conserved}

Findings described above in this section strongly support the notion that the mechanisms by which phytochemicals extend longevity of various heterotrophic organisms have been conserved in the course of evolution. Indeed, longevity-extending phytochemicals increase lifespan of such evolutionarily distant organisms as yeasts, worms, flies, bees, mosquitoes, fishes, laboratory mice, and laboratory rats $[17,61-78,80-87,89-95,97-101,103-109,111,112,115]$; these phytochemicals also prolong the replicative lifespans of different lines of cultured human cells [79,88,96,102,111] (Table 1). Furthermore, lifespan-prolonging abilities of these phytochemicals rely on cellular proteins integrated into several evolutionarily conserved signaling pathways known to regulate longevity in organisms across phyla [52,117-126]. These nutrient-, energy- and stress-sensing pathways include the following: (1) the IIS pathway $[67,70-72,74,80,81,86,89,90,99-101,114,115]$; (2) the TOR pathway $[17,68,69]$; (3) the sirtuin-governed protein deacetylation module of the longevity signaling network integrating the IIS and TOR pathways [45,54,60,66,67,74,106,109]; (4) the OSR-1/UNC-43 (CaMKII)/SEK-1 (p38 MAPK) stress-responsive signaling pathway [67,74,101]; and (5) the non-selective autophagy pathway for degradation of various cellular organelles and macromolecules [111-113] (Table 1). Moreover, these lifespan-prolonging phytochemicals postpone the onset of several longevity-defining cellular processes called "the cellular and molecular hallmarks of aging" [52,117,119,121,122,124,127-133]. Out of the nine commonly accepted cellular and molecular hallmarks of aging [124], lifespan-prolonging 
phytochemicals are known to delay the development of the following seven common traits of aging in evolutionarily distant heterotrophic organisms: (1) genomic instability [66]; (2) epigenetic alterations [111]; (3) loss of proteostasis [67,68,70,71,73-77,79,81-86,88-91,96,98,102,105,110,111,115]; (4) deregulated nutrient sensing $\quad[67,70,71,74,86,89,109]$; (5) mitochondrial dysfunction [17,72,74,82,83,86,87,90,94,96-98,102,109]; (6) cellular senescence [62-65,69,88,102]; and (7) altered intercellular communication [62-65,73,78,107,112] (Table 1).

\section{Phytochemicals: Interspecies Chemical Signals That May Contribute to the Evolution of Longevity Regulation Mechanisms within Natural Ecosystems}

Findings summarized in the previous section provided the comprehensive evidence that many phytochemicals can extend lifespans of heterotrophic organisms across phyla via evolutionarily conserved mechanisms. These findings gave rise to a hypothesis on how such lifespan-extending capabilities of phytochemicals may contribute to the evolution of longevity regulation mechanisms in various organisms inhabiting a natural ecosystem. In this section, we discuss and extend this hypothesis.

\subsection{The "Xenohormesis" Hypothesis}

The term "hormesis" has been introduced to define a special kind of response of cells and organisms to different doses of a stress agent. In this kind of stress response: (1) an exposure of a cell or an organism to low ("hormetic") doses of a stress agent stimulates its growth, proliferation and/or survival; whereas (2) high doses of the same stress agent exhibit adverse effects on growth, proliferation and/or survival of this cell or organism [134-137]. Graphically, hormetic stress response is defined by a nonlinear and biphasic dose-response curve, which could be U-shaped, inverted U-shaped or J-shaped [44,57,138,139]. It is commonly accepted that an exposure of a cell or an organism to low doses of a hormetic stress agent elicits certain adaptive changes; by preconditioning the cell or the organism to a moderate stress, such changes can help to protect it against higher doses of the same (or related) stress agent [44,57,138-140].

The xenohormesis hypothesis posits that plants synthesize phytochemicals, in part, as a response to such hormetic environmental stresses as UV light, heat and cold stresses, osmotic stress and high salinity, water deficit and dehydration, nutrient deprivation, and infection [66,141,142]. Within the plant that synthesizes such phytochemicals, these secondary metabolites present at the concentrations that are not toxic but create a mild stress. Thus, within the host plant, such phytochemicals function as hormetic stress agents capable of inducing certain defense systems; these systems protect the plant against higher doses of the environmental stress that caused the synthesis of the phytochemicals and, possibly, against related environmental stresses [66,141,142]. According to the xenohormesis hypothesis, after the plant has released the phytochemicals into the ecosystem, these secondary metabolites (1) do not have any hormetic effect on the heterotrophic organisms inhabiting the ecosystem (and, thus, act as hormetic stress agents only within the host plant) — likely because the concentration of the phytochemicals outside the plant is below a hormetic threshold; (2) provide the heterotrophic organisms within the ecosystem with chemically encoded information on various changes in the environmental conditions taking place in the ecosystem; and (3) operate as interspecies chemical signals that can extend lifespans of various heterotrophic organisms within the ecosystem via evolutionarily conserved mechanisms, as described in the previous section $[66,141,142]$. The xenohormesis hypothesis postulates that the ability of 
heterotrophic organisms to remodel their metabolism and physiology in response to phytochemicals as messages on environmental changes within the ecosystem will increase their chances of survival, thus creating selective forces aimed at maintaining such ability [66,141,142]. Because many of these phytochemicals are also known to extend longevity in heterotrophic organisms across phyla by targeting evolutionarily conserved mechanisms, these selective forces may provide a mode of selection for the most efficient longevity regulation mechanisms [66,141,142]. Thus, phytochemicals may function as xenohormetic stress signals that drive the ecosystemic evolution of such mechanisms in heterotrophic organisms.

\subsection{Many Observations Contradict the Xenohormesis Hypothesis}

One of the predictions of the xenohormesis hypothesis is that phytochemicals do not act as hormetic stress agents in heterotrophic organisms inhabiting the ecosystem [66,141,142]. However, recent findings imply that the dose-response effect of some phytochemicals on the longevity of heterotrophic organisms can be graphically described as an inverted U-shaped curve; these phytochemicals include caffeic acid, caffeine, ellagic acid, epigallocatechin gallate, quercetin, rosmarinic acid, and tannic acid $[43,44,67,71,81,141]$. Thus, at least caffeic acid, caffeine, ellagic acid, epigallocatechin gallate, quercetin, rosmarinic acid, and tannic acid can elicit a hormetic stress response in heterotrophic organisms; these phytochemicals are likely to operate as hormetic stress agents within both the host plants synthesizing them and heterotrophic organisms exposed to them.

Furthermore, some phytochemicals exhibit moderate cytostatic effects in heterotrophic organisms across phyla. These phytochemicals include resveratrol and caffeine; they both act as cytostatic agents that retard cellular and organismal growth because of their abilities to attenuate the pro-aging TOR signaling pathway, a key driver of proliferative growth in heterotrophic organisms [68,126,143-149].

Moreover, some phytochemicals are not secondary metabolites that exist at low concentrations and are synthesized in secondary biochemical pathways, but primary metabolites that are relatively abundant and synthesized in primary biochemical pathways - one example of such phytochemicals is unsaturated fatty acids synthesized by plants in response to cold stress [13,150,151]. After being consumed by mammals, these unsaturated fatty acids are incorporated into cellular membranes in substantial quantities, thereby increasing membrane fluidity; this, in turn, elicits a longevity-extending process of heat shock response aimed at enhancing such age-delaying process as proteostasis maintenance $[13,152,153]$.

\subsection{An Extended Hypothesis on the Role of Phytochemicals in the Ecosystemic Evolution of Longevity} Regulation Mechanisms

Because of the above contradictions to some projections of the xenohormesis hypothesis, we extend it by proposing a hypothesis in which phytochemicals that have been released by plants into a natural ecosystem may create xenohormetic, hormetic and cytostatic selective forces driving the evolution of longevity regulation mechanisms in heterotrophic organisms within this ecosystem. Our extended hypothesis posits that phytochemicals creating such selective forces after being released by plants into the ecosystem: (1) are either low-abundance chemical compounds formed by plants in secondary biochemical pathways or high-abundance products of primary biochemical pathways; (2) do not act as hormetic stress agents in heterotrophic organisms inhabiting the ecosystem or, alternatively, can trigger 
hormetic stress response in these organisms; (3) do not slow down growth of heterotrophic organisms within the ecosystem or, alternatively, cause a moderate delay of such growth by attenuating the pro-aging TOR signaling pathway; and (4) can increase lifespans of heterotrophic organisms inhabiting the ecosystem by modulating several evolutionarily conserved signaling pathways regulating longevity in these organisms; these signaling pathways and mechanisms of their modulation by phytochemicals have been described in the previous section.

\section{Conclusions and Future Perspectives}

Growing evidence supports the view that phytochemicals prolong lifespan in heterotrophic organisms across phyla by modulating a complex network of evolutionarily conserved signaling pathways; these pathways orchestrate a compendium of longevity-defining cellular processes that have been conserved in the course of evolution. The molecular and cellular mechanisms by which structurally diverse phytochemicals extend longevity of various heterotrophic organisms have emerged. Based on these findings, a hypothesis has been proposed on how the release of phytochemicals by plants into a natural ecosystem may create selective forces that guide the evolution of longevity regulation mechanisms in heterotrophic organisms inhabiting this ecosystem. The major challenge now is to empirically validate this hypothesis, perhaps by conducting experimental evolution of longevity regulation mechanisms in naturally short-lived heterotrophic organisms (such as the yeast $S$. cerevisiae, nematode $C$. elegans, fruit fly D. melanogaster, and fish $N$. furzeri) exposed to phytochemicals under laboratory conditions that mimic the natural stressful environment of cyclical starvation. It will be interesting to see if such long-term exposure of these organisms to phytochemicals can lead to selection of species that live longer than their predecessors. If such laboratory-evolved long-lived species can be selected, it will be intriguing to measure the relative fitness of each of them in a direct competition assay with their relatively short-lived ancestors. These experiments will provide an empirical validation test of the numerous evolutionary theories of aging trying to explain how the evolutionary force actively limits organismal lifespan at an age unique to each species [154-163].

\section{Acknowledgments}

We are grateful to current and former members of the Titorenko laboratory for discussions. This research was supported by grants from the NSERC of Canada and Concordia University Chair Fund to Vladimir I. Titorenko. Amanda Piano was supported by a Frederick Banting and Charles Best Canada Master's Scholarship Award from the CIHR. Veronika Svistkova was supported by an Undergraduate Summer Award from the NSERC of Canada. Vladimir I. Titorenko is a Concordia University Research Chair in Genomics, Cell Biology and Aging.

\section{Author Contributions}

Authors contributed equally to the preparation of this review.

\section{Conflicts of Interest}

The authors declare no conflict of interest. 


\section{References}

1. Harborne, J.R. Introduction to Ecological Biochemistry, 4th ed.; Elsevier Inc.: London, UK, 1993; p. 316.

2. Gershenzon, J. The cost of plant chemical defense against herbivory: A biochemical perspective. In Insect-Plant Interactions; Bernays, E.A., Ed.; CRC Press: Boca Raton, USA, 1994; pp. 105-173.

3. Reymond, P.; Weber, H.; Damond, M.; Farmer, E.E. Differential gene expression in response to mechanical wounding and insect feeding in Arabidopsis. Plant Cell 2000, 12, 707-720.

4. Hermsmeier, D.; Schittko, U.; Baldwin, I.T. Molecular interactions between the specialist herbivore Manduca sexta (Lepidoptera, Sphingidae) and its natural host Nicotiana attenuata. I. Large-scale changes in the accumulation of growth- and defense-related plant mRNAs. Plant Physiol. 2001, $125,683-700$.

5. Kennedy, D.O.; Wightman, E.L. Herbal extracts and phytochemicals: Plant secondary metabolites and the enhancement of human brain function. Adv. Nutr. 2011, 2, 32-50.

6. Strobel, G.; Daisy, B.; Castillo, U.; Harper, J. Natural products from endophytic microorganisms. J. Nat. Prod. 2004, 67, 257-268.

7. Verma, V.C.; Kharwar, R.N.; Strobel, G.A. Chemical and functional diversity of natural products from plant associated endophytic fungi. Nat. Prod. Commun. 2009, 4, 1511-1532.

8. Yu, H.; Zhang, L.; Li, L.; Zheng, C.; Guo, L.; Li, W.; Sun, P.; Qin, L. Recent developments and future prospects of antimicrobial metabolites produced by endophytes. Microbiol. Res. 2010, 165, 437-449.

9. Bascom-Slack, C.A.; Arnold, A.E.; Strobel, S.A. IBI series winner. Student-directed discovery of the plant microbiome and its products. Science 2012, 338, 485-486.

10. Aly, A.H.; Debbab, A.; Proksch, P. Fungal endophytes-Secret producers of bioactive plant metabolites. Pharmazie 2013, 68, 499-505.

11. Mousa, W.K.; Raizada, M.N. The diversity of anti-microbial secondary metabolites produced by fungal endophytes: An interdisciplinary perspective. Front. Microbiol. 2013, 4, 65.

12. Hansen, B.G.; Halkier, B.A. New insight into the biosynthesis and regulation of indole compounds in Arabidopsis thaliana. Planta 2005, 221, 603-606.

13. Hooper, P.L.; Hooper, P.L.; Tytell, M.; Vígh, L. Xenohormesis: Health benefits from an eon of plant stress response evolution. Cell Stress Chaperones 2010, 15, 761-770.

14. Higdon, J.; Drake, V.J. An Evidence-Based Approach to Phytochemicals and Other Dietary Factors, 2nd ed.; Thieme: New York, NY, USA, 2012; p. 328.

15. Menendez, J.A.; Joven, J.; Aragonès, G.; Barrajón-Catalán, E.; Beltrán-Debón, R.; Borrás-Linares, I.; Camps, J.; Corominas-Faja, B.; Cufí, S.; Fernández-Arroyo, S.; et al. Xenohormetic and anti-aging activity of secoiridoid polyphenols present in extra virgin olive oil: A new family of gerosuppressant agents. Cell Cycle 2013, 12, 555-578.

16. Si, H.; Liu, D. Dietary antiaging phytochemicals and mechanisms associated with prolonged survival. J. Nutr. Biochem. 2014, 25, 581-591.

17. Wu, Z.; Song, L.; Liu, S.Q.; Huang, D. Tanshinones extend chronological lifespan in budding yeast Saccharomyces cerevisiae. Appl. Microbiol. Biotechnol. 2014, 98, 8617-8628. 
18. Somani, S.J.; Modi, K.P.; Majumdar, A.S.; Sadarani, B.N. Phytochemicals and their potential usefulness in inflammatory bowel disease. Phytother. Res. 2015, 29, 339-350.

19. Wink, M. Evolution of secondary metabolites from an ecological and molecular phylogenetic perspective. Phytochemistry 2003, 64, 3-19.

20. Tahara, S. A journey of twenty-five years through the ecological biochemistry of flavonoids. Biosci. Biotechnol. Biochem. 2007, 71, 1387-1404.

21. Murakami, A. Modulation of protein quality control systems by food phytochemicals. J. Clin. Biochem. Nutr. 2013, 52, 215-227.

22. Adrian, M.; Jeandet, P.; Veneau, J.; Weston, L.A.; Bessis, R. Biological activity of resveratrol, a stilbenic compound from grapevines, against Botrytis cinerea, the causal agent for gray mold. J. Chem. Ecol. 1997, 23, 1689-1702.

23. Heath, M.C. Hypersensitive response-related death. Plant Mol. Biol. 2000, 44, 321-334.

24. Trewavas, A.; Stewart, D. Paradoxical effects of chemicals in the diet on health. Curr. Opin. Plant Biol. 2003, 6, 185-190.

25. Arimura, G.; Kost, C.; Boland, W. Herbivore-induced, indirect plant defences. Biochim. Biophys. Acta 2005, 1734, 91-111.

26. Brencic, A.; Winans, S.C. Detection of and response to signals involved in host-microbe interactions by plant-associated bacteria. Microbiol. Mol. Biol. Rev. 2005, 69, 155-194.

27. Mattson, M.P.; Son, T.G.; Camandola, S. Viewpoint: Mechanisms of action and therapeutic potential of neurohormetic phytochemicals. Dose Response 2007, 5, 174-186.

28. Santiago, R.; Malvar, R.A. Role of dehydrodiferulates in maize resistance to pests and diseases. Int. J. Mol. Sci. 2010, 11, 691-703.

29. Tang, K.; Zhan, J.C.; Yang, H.R.; Huang, W.D. Changes of resveratrol and antioxidant enzymes during UV-induced plant defense response in peanut seedlings. J. Plant Physiol. 2010, 167, 95-102.

30. Arimura, G.; Ozawa, R.; Maffei, M.E. Recent advances in plant early signaling in response to herbivory. Int. J. Mol. Sci. 2011, 12, 3723-3739.

31. Barros-Rios, J.; Malvar, R.A.; Jung, H.J.; Santiago, R. Cell wall composition as a maize defense mechanism against corn borers. Phytochemistry 2011, 72, 365-371.

32. Bednarek, P. Sulfur-containing secondary metabolites from Arabidopsis thaliana and other Brassicaceae with function in plant immunity. ChemBioChem 2012, 13, 1846-1859.

33. Nwachukwu, I.D.; Slusarenko, A.J.; Gruhlke, M.C. Sulfur and sulfur compounds in plant defence. Nat. Prod. Commun. 2012, 7, 395-400.

34. Huot, O.B.; Nachappa, P.; Tamborindeguy, C. The evolutionary strategies of plant defenses have a dynamic impact on the adaptations and interactions of vectors and pathogens. Insect Sci. 2013, 20, 297-306.

35. Kazan, K.; Lyons, R. Intervention of phytohormone pathways by pathogen effectors. Plant Cell 2014, 26, 2285-2309.

36. Porras-Alfaro, A.; Bayman, P. Hidden fungi, emergent properties: Endophytes and microbiomes. Annu. Rev. Phytopathol. 2011, 49, 291-315.

37. Zhao, J.; Shan, T.; Mou, Y.; Zhou, L. Plant-derived bioactive compounds produced by endophytic fungi. Mini Rev. Med. Chem. 2011, 11, 159-168. 
38. Kusari, S.; Hertweck, C.; Spiteller, M. Chemical ecology of endophytic fungi: Origins of secondary metabolites. Chem. Biol. 2012, 19, 792-798.

39. Nath, A.; Raghunatha, P.; Joshi, S.R. Diversity and biological activities of endophytic fungi of Emblica officinalis, an ethnomedicinal plant of India. Mycobiology 2012, 40, 8-13.

40. Zhang, Y.; Han, T.; Ming, Q.; Wu, L.; Rahman, K.; Qin, L. Alkaloids produced by endophytic fungi: A review. Nat. Prod. Commun. 2012, 7, 963-968.

41. Lebeis, S.L. The potential for give and take in plant-microbiome relationships. Front. Plant Sci. 2014, 5, 287.

42. Harikumar, K.B.; Aggarwal, B.B. Resveratrol: A multitargeted agent for age-associated chronic diseases. Cell Cycle 2008, 7, 1020-1035.

43. Son, T.G.; Camandola, S.; Mattson, M.P. Hormetic dietary phytochemicals. Neuromol. Med. 2008, 10, 236-246.

44. Calabrese, V.; Cornelius, C.; Dinkova-Kostova, A.T.; Iavicoli, I.; Di Paola, R.; Koverech, A.; Cuzzocrea, S.; Rizzarelli, E.; Calabrese, E.J. Cellular stress responses, hormetic phytochemicals and vitagenes in aging and longevity. Biochim. Biophys. Acta 2012, 1822, 753-783.

45. Dong, Y.; Guha, S.; Sun, X.; Cao, M.; Wang, X.; Zou, S. Nutraceutical interventions for promoting healthy aging in invertebrate models. Oxid. Med. Cell. Longev. 2012, 2012, 718491.

46. Lamming, D.W.; Sabatini, D.M.; Baur, J.A. Pharmacologic means of extending lifespan. J. Clin. Exp. Pathol. 2012, 4, 7327.

47. Vauzour, D. Dietary polyphenols as modulators of brain functions: Biological actions and molecular mechanisms underpinning their beneficial effects. Oxid. Med. Cell. Longev. 2012, 2012, 914273.

48. Argyropoulou, A.; Aligiannis, N.; Trougakos, I.P.; Skaltsounis, A.L. Natural compounds with anti-ageing activity. Nat. Prod. Rep. 2013, 30, 1412-1437.

49. Lee, J.H.; Khor, T.O.; Shu, L.; Su, Z.Y.; Fuentes, F.; Kong, A.N. Dietary phytochemicals and cancer prevention: Nrf2 signaling, epigenetics, and cell death mechanisms in blocking cancer initiation and progression. Pharmacol. Ther. 2013, 137, 153-171.

50. Lucanic, M.; Lithgow, G.J.; Alavez, S. Pharmacological lifespan extension of invertebrates. Ageing Res. Rev. 2013, 12, 445-458.

51. Monroy, A.; Lithgow, G.J.; Alavez, S. Curcumin and neurodegenerative diseases. Biofactors 2013, 39, 122-132.

52. De Cabo, R.; Carmona-Gutierrez, D.; Bernier, M.; Hall, M.N.; Madeo, F. The search for antiaging interventions: From elixirs to fasting regimens. Cell 2014, 157, 1515-1526.

53. Grabacka, M.M.; Gawin, M.; Pierzchalska, M. Phytochemical modulators of mitochondria: The search for chemopreventive agents and supportive therapeutics. Pharmaceuticals 2014, 7 , 913-942.

54. Hubbard, B.P.; Sinclair, D.A. Small molecule SIRT1 activators for the treatment of aging and age-related diseases. Trends Pharmacol. Sci. 2014, 35, 146-154.

55. Kennedy, D.O. Polyphenols and the human brain: Plant "secondary metabolite" ecologic roles and endogenous signaling functions drive benefits. Adv. Nutr. 2014, 5, 515-533.

56. Koch, K.; Havermann, S.; Büchter, C.; Wätjen, W. Caenorhabditis elegans as model system in pharmacology and toxicology: Effects of flavonoids on redox-sensitive signalling pathways and ageing. ScientificWorldJournal 2014, 2014, 920398. 
57. Lee, J.; Jo, D.G.; Park, D.; Chung, H.Y.; Mattson, M.P. Adaptive cellular stress pathways as therapeutic targets of dietary phytochemicals: Focus on the nervous system. Pharmacol. Rev. 2014, 66, 815-868.

58. Mansuri, M.L.; Parihar, P.; Solanki, I.; Parihar, M.S. Flavonoids in modulation of cell survival signalling pathways. Genes Nutr. 2014, 9, 400.

59. Rege, S.D.; Geetha, T.; Griffin, G.D.; Broderick, T.L.; Babu, J.R. Neuroprotective effects of resveratrol in Alzheimer disease pathology. Front. Aging Neurosci. 2014, 6, 218.

60. Sinclair, D.A.; Guarente, L. Small-molecule allosteric activators of sirtuins. Annu. Rev. Pharmacol. Toxicol. 2014, 54, 363-380.

61. Pan, W.; Jiang, S.; Luo, P.; Wu, J.; Gao, P. Isolation, purification and structure identification of antioxidant compound from the roots of Incarvillea younghusbandii Sprague and its life span prolonging effect in Drosophila melanogaster. Nat. Prod. Res. 2008, 22, 719-725.

62. Moriguchi, T.; Takashina, K.; Chu, P.J.; Saito, H.; Nishiyama, N. Prolongation of life span and improved learning in the senescence accelerated mouse produced by aged garlic extract. Biol. Pharm. Bull. 1994, 17, 1589-1594.

63. Moriguchi, T.; Saito, H.; Nishiyama, N. Aged garlic extract prolongs longevity and improves spatial memory deficit in senescence-accelerated mouse. Biol. Pharm. Bull. 1996, 19, 305-307.

64. Moriguchi, T.; Saito, H.; Nishiyama, N. Anti-ageing effect of aged garlic extract in the inbred brain atrophy mouse model. Clin. Exp. Pharmacol. Physiol. 1997, 24, 235-242.

65. Nishiyama, N.; Moriguchi, T.; Saito, H. Beneficial effects of aged garlic extract on learning and memory impairment in the senescence-accelerated mouse. Exp. Gerontol. 1997, 32, 149-160.

66. Howitz, K.T.; Bitterman, K.J.; Cohen, H.Y.; Lamming, D.W.; Lavu, S.; Wood, J.G.; Zipkin, R.E.; Chung, P.; Kisielewski, A.; Zhang, L.L.; Scherer, B.; Sinclair, D.A. Small molecule activators of sirtuins extend Saccharomyces cerevisiae lifespan. Nature 2003, 425, 191-196.

67. Pietsch, K.; Saul, N.; Chakrabarti, S.; Stürzenbaum, S.R.; Menzel, R.; Steinberg, C.E. Hormetins, antioxidants and prooxidants: Defining quercetin-, caffeic acid- and rosmarinic acid-mediated life extension in C. elegans. Biogerontology 2011, 12, 329-347.

68. Wanke, V.; Cameroni, E.; Uotila, A.; Piccolis, M.; Urban, J.; Loewith, R.; De Virgilio, C. Caffeine extends yeast lifespan by targeting TORC1. Mol. Microbiol. 2008, 69, 277-285.

69. Rallis, C.; Codlin, S.; Bähler, J. TORC1 signaling inhibition by rapamycin and caffeine affect lifespan, global gene expression, and cell proliferation of fission yeast. Aging Cell 2013, 12, 563-573.

70. Lublin, A.; Isoda, F.; Patel, H.; Yen, K.; Nguyen, L.; Hajje, D.; Schwartz, M.; Mobbs, C. FDA-approved drugs that protect mammalian neurons from glucose toxicity slow aging dependent on Cbp and protect against proteotoxicity. PLOS ONE 2011, 6, e27762.

71. Sutphin, G.L.; Bishop, E.; Yanos, M.E.; Moller, R.M.; Kaeberlein, M. Caffeine extends life span, improves healthspan, and delays age-associated pathology in Caenorhabditis elegans. Longev. Healthspan 2012, 1, 9.

72. Saul, N.; Pietsch, K.; Menzel, R.; Stürzenbaum, S.R.; Steinberg, C.E. Catechin induced longevity in C. elegans: From key regulator genes to disposable soma. Mech. Ageing Dev. 2009, 130, 477-486.

73. Kiaei, M.; Kipiani, K.; Petri, S.; Chen, J.; Calingasan, N.Y.; Beal, M.F. Celastrol blocks neuronal cell death and extends life in transgenic mouse model of amyotrophic lateral sclerosis. Neurodegener. Dis. 2005, 2, 246-254. 
74. Liao, V.H.; Yu, C.W.; Chu, Y.J.; Li, W.H.; Hsieh, Y.C.; Wang, T.T. Curcumin-mediated lifespan extension in Caenorhabditis elegans. Mech. Ageing Dev. 2011, 132, 480-487.

75. Lee, K.S.; Lee, B.S.; Semnani, S.; Avanesian, A.; Um, C.Y.; Jeon, H.J.; Seong, K.M.; Yu, K.; Min, K.J.; Jafari, M. Curcumin extends life span, improves health span, and modulates the expression of age-associated aging genes in Drosophila melanogaster. Rejuvenation Res. 2010 , 13, 561-570.

76. Shen, L.R.; Xiao, F.; Yuan, P.; Chen, Y.; Gao, Q.K.; Parnell, L.D.; Meydani, M.; Ordovas, J.M.; Li, D.; Lai, C.Q. Curcumin-supplemented diets increase superoxide dismutase activity and mean lifespan in Drosophila. Age 2013, 35, 1133-1142.

77. Caesar, I.; Jonson, M.; Nilsson, K.P.; Thor, S.; Hammarström, P. Curcumin promotes A-beta fibrillation and reduces neurotoxicity in transgenic Drosophila. PLoS ONE 2012, 7, e31424.

78. Bakshi, H.A.; Sam, S.; Feroz, A.; Ravesh, Z.; Shah, G.A.; Sharma, M. Crocin from Kashmiri saffron (Crocus sativus) induces in vitro and in vivo xenograft growth inhibition of Dalton's lymphoma (DLA) in mice. Asian Pac. J. Cancer Prev. 2009, 10, 887-890.

79. Choi, M.J.; Kim, B.K.; Park, K.Y.; Yokozawa, T.; Song, Y.O.; Cho, E.J. Anti-aging effects of cyanidin under a stress-induced premature senescence cellular system. Biol. Pharm. Bull. 2010, 33, 421-426.

80. Powolny, A.A.; Singh, S.V.; Melov, S.; Hubbard, A.; Fisher, A.L. The garlic constituent diallyl trisulfide increases the lifespan of C. elegans via skn-1 activation. Exp. Gerontol. 2011, 46, 441-452.

81. Saul, N.; Pietsch, K.; Stürzenbaum, S.R.; Menzel, R.; Steinberg, C.E. Diversity of polyphenol action in Caenorhabditis elegans: Between toxicity and longevity. J. Nat. Prod. 2011, 74, 1713-1720.

82. Abbas, S.; Wink, M. Epigallocatechin gallate from green tea (Camellia sinensis) increases lifespan and stress resistance in Caenorhabditis elegans. Planta Med. 2009, 75, 216-221.

83. Abbas, S.; Wink, M. Epigallocatechin gallate inhibits beta amyloid oligomerization in Caenorhabditis elegans and affects the daf-2/insulin-like signaling pathway. Phytomedicine 2010, 17, 902-909.

84. Si, H.; Fu, Z.; Babu, P.V.; Zhen, W.; Leroith, T.; Meaney, M.P.; Voelker, K.A.; Jia, Z.; Grange, R.W.; Liu, D. Dietary epicatechin promotes survival of obese diabetic mice and Drosophila melanogaster. J. Nutr. 2011, 141, 1095-1100.

85. Sayed, A.A. Ferulsinaic acid attenuation of advanced glycation end products extends the lifespan of Caenorhabditis elegans. J. Pharm. Pharmacol. 2011, 63, 423-428.

86. Kampkötter, A.; Nkwonkam, C.G.; Zurawski, R.F.; Timpel, C.; Chovolou, Y.; Wätjen, W.; Kahl, R. Effects of the flavonoids kaempferol and fisetin on thermotolerance, oxidative stress and FoxO transcription factor DAF-16 in the model organism Caenorhabditis elegans. Arch. Toxicol. 2007, 81, 849-858.

87. Zarse, K.; Bossecker, A.; Müller-Kuhrt, L.; Siems, K.; Hernandez, M.A.; Berendsohn, W.G.; Birringer, M.; Ristow, M. The phytochemical glaucarubinone promotes mitochondrial metabolism, reduces body fat, and extends lifespan of Caenorhabditis elegans. Horm. Metab. Res. 2011, 43, 241-243. 
88. Wang, P.; Zhang, Z.; Ma, X.; Huang, Y.; Liu, X.; Tu, P.; Tong, T. HDTIC-1 and HDTIC-2, two compounds extracted from Astragali Radix, delay replicative senescence of human diploid fibroblasts. Mech. Ageing Dev. 2003, 124, 1025-1034.

89. Cai, W.J.; Huang, J.H.; Zhang, S.Q.; Wu, B.; Kapahi, P.; Zhang, X.M.; Shen, Z.Y. Icariin and its derivative icariside II extend healthspan via insulin/IGF-1 pathway in C. elegans. PLoS ONE 2011, 6, e28835.

90. Grünz, G.; Haas, K.; Soukup, S.; Klingenspor, M.; Kulling, S.E.; Daniel, H.; Spanier, B. Structural features and bioavailability of four flavonoids and their implications for lifespan-extending and antioxidant actions in C. elegans. Mech. Ageing Dev. 2012, 133, 1-10.

91. West, M.; Mhatre, M.; Ceballos, A.; Floyd, R.A.; Grammas, P.; Gabbita, S.P.; Hamdheydari, L.; Mai, T.; Mou, S.; Pye, Q.N.; et al. The arachidonic acid 5-lipoxygenase inhibitor nordihydroguaiaretic acid inhibits tumor necrosis factor alpha activation of microglia and extends survival of G93A-SOD1 transgenic mice. J. Neurochem. 2004, 91, 133-143.

92. Strong, R.; Miller, R.A.; Astle, C.M.; Floyd, R.A.; Flurkey, K.; Hensley, K.L.; Javors, M.A.; Leeuwenburgh, C.; Nelson, J.F.; Ongini, E.; et al. Nordihydroguaiaretic acid and aspirin increase lifespan of genetically heterogeneous male mice. Aging Cell 2008, 7, 641-650.

93. Buu-Hoi, N.P.; Ratsimamanga, A.R. Retarding action of nordihydroguaiaretic acid on aging in the rat. C. R. Seances Soc. Biol. Fil. 1959, 153, 1180-1182.

94. Miquel, J.; Fleming, J.; Economos, A.C. Antioxidants, metabolic rate and aging in Drosophila. Arch. Gerontol. Geriatr. 1982, 1, 159-165.

95. Richie, J.P., Jr.; Mills, B.J.; Lang, C.A. Dietary nordihydroguaiaretic acid increases the life span of the mosquito. Proc. Soc. Exp. Biol. Med. 1986, 183, 81-85.

96. Katsiki, M.; Chondrogianni, N.; Chinou, I.; Rivett, A.J.; Gonos, E.S. The olive constituent oleuropein exhibits proteasome stimulatory properties in vitro and confers life span extension of human embryonic fibroblasts. Rejuvenation Res. 2007, 10, 157-172.

97. Xiang, L.; Sun, K.; Lu, J.; Weng, Y.; Taoka, A.; Sakagami, Y.; Qi, J. Anti-aging effects of phloridzin, an apple polyphenol, on yeast via the SOD and Sir2 genes. Biosci. Biotechnol. Biochem. 2011, 75, 854-858.

98. Belinha, I.; Amorim, M.A.; Rodrigues, P.; de Freitas, V.; Moradas-Ferreira, P.; Mateus, N.; Costa, V. Quercetin increases oxidative stress resistance and longevity in Saccharomyces cerevisiae. J. Agric. Food Chem. 2007, 55, 2446-2451.

99. Kampkötter, A.; Nkwonkam, C.G.; Zurawski, R.F.; Timpel, C.; Chovolou, Y.; Wätjen, W.; Kahl, R. Investigations of protective effects of the flavonoids quercetin and rutin on stress resistance in the model organism Caenorhabditis elegans. Toxicology 2007, 234, 113-123.

100. Kampkötter, A.; Timpel, C.; Zurawski, R.F.; Ruhl, S.; Chovolou, Y.; Proksch, P.; Wätjen, W. Increase of stress resistance and lifespan of Caenorhabditis elegans by quercetin. Comp. Biochem. Physiol. B Biochem. Mol. Biol. 2008, 149, 314-323.

101. Pietsch, K.; Saul, N.; Menzel, R.; Stürzenbaum, S.R.; Steinberg, C.E. Quercetin mediated lifespan extension in Caenorhabditis elegans is modulated by age-1, daf-2, sek-1 and unc-43. Biogerontology 2009, 10, 565-578.

102. Chondrogianni, N.; Kapeta, S.; Chinou, I.; Vassilatou, K.; Papassideri, I.; Gonos, E.S. Anti-ageing and rejuvenating effects of quercetin. Exp. Gerontol. 2010, 45, 763-771. 
103. Xue, Y.L.; Ahiko, T.; Miyakawa, T.; Amino, H.; Hu, F.; Furihata, K.; Kita, K.; Shirasawa, T.; Sawano, Y.; Tanokura, M. Isolation and Caenorhabditis elegans lifespan assay of flavonoids from onion. J. Agric. Food Chem. 2011, 59, 5927-5934.

104. Srivastava, D.; Arya, U.; SoundaraRajan, T.; Dwivedi, H.; Kumar, S.; Subramaniam, JR. Reserpine can confer stress tolerance and lifespan extension in the nematode C. elegans. Biogerontology 2008, 9, 309-316.

105. Arya, U.; Dwivedi, H.; Subramaniam, J.R. Reserpine ameliorates Abeta toxicity in the Alzheimer's disease model in Caenorhabditis elegans. Exp. Gerontol. 2009, 44, 462-466.

106. Wood, J.G.; Rogina, B.; Lavu, S.; Howitz, K.; Helfand, S.L.; Tatar, M.; Sinclair, D. Sirtuin activators mimic caloric restriction and delay ageing in metazoans. Nature 2004, 430, 686-689.

107. Valenzano, D.R.; Terzibasi, E.; Genade, T.; Cattaneo, A.; Domenici, L.; Cellerino, A. Resveratrol prolongs lifespan and retards the onset of age-related markers in a short-lived vertebrate. Curr. Biol. 2006, 16, 296-300.

108. Rascón, B.; Hubbard, B.P.; Sinclair, D.A.; Amdam, G.V. The lifespan extension effects of resveratrol are conserved in the honey bee and may be driven by a mechanism related to caloric restriction. Aging 2012, 4, 499-508.

109. Baur, J.A.; Pearson, K.J.; Price, N.L.; Jamieson, H.A.; Lerin, C.; Kalra, A.; Prabhu, V.V.; Allard, J.S.; Lopez-Lluch, G.; Lewis, K.; et al. Resveratrol improves health and survival of mice on a high-calorie diet. Nature 2006, 444, 337-342.

110. Morselli, E.; Maiuri, M.C.; Markaki, M.; Megalou, E.; Pasparaki, A.; Palikaras, K.; Criollo, A.; Galluzzi, L.; Malik, S.A.; Vitale, I.; et al. Caloric restriction and resveratrol promote longevity through the Sirtuin-1-dependent induction of autophagy. Cell Death Dis. 2010, 1, e10.

111. Eisenberg, T.; Knauer, H.; Schauer, A.; Büttner, S.; Ruckenstuhl, C.; Carmona-Gutierrez, D.; Ring, J.; Schroeder, S.; Magnes, C.; Antonacci, L.; et al. Induction of autophagy by spermidine promotes longevity. Nat. Cell Biol. 2009, 11, 1305-1314.

112. Minois, N.; Carmona-Gutierrez, D.; Bauer, M.A.; Rockenfeller, P.; Eisenberg, T.; Brandhorst, S.; Sigrist, S.J.; Kroemer, G.; Madeo, F. Spermidine promotes stress resistance in Drosophila melanogaster through autophagy-dependent and-independent pathways. Cell Death Dis. 2012, 3, e401.

113. Minois, N.; Rockenfeller, P.; Smith, T.K.; Carmona-Gutierrez, D. Spermidine feeding decreases age-related locomotor activity loss and induces changes in lipid composition. PLOS ONE 2014, 9, e102435.

114. Saul, N.; Pietsch, K.; Menzel, R.; Stürzenbaum, S.R.; Steinberg, C.E. The longevity effect of tannic acid in Caenorhabditis elegans: Disposable Soma meets hormesis. J. Gerontol. A Biol. Sci. Med. Sci. 2010, 65, 626-635.

115. Cañuelo, A.; Gilbert-López, B.; Pacheco-Liñán, P.; Martínez-Lara, E.; Siles, E.; Miranda-Vizuete, A. Tyrosol, a main phenol present in extra virgin olive oil, increases lifespan and stress resistance in Caenorhabditis elegans. Mech. Ageing Dev. 2012, 133, 563-574.

116. Strong, R.; Miller, R.A.; Astle, C.M.; Baur, J.A.; de Cabo, R.; Fernandez, E.; Guo, W.; Javors, M.; Kirkland, J.L.; Nelson, J.F.; et al. Evaluation of resveratrol, green tea extract, curcumin, oxaloacetic acid, and medium-chain triglyceride oil on life span of genetically heterogeneous mice. J. Gerontol. A Biol. Sci. Med. Sci. 2013, 68, 6-16. 
117. Fontana, L.; Partridge, L.; Longo, V.D. Extending healthy life span-from yeast to humans. Science 2010, 328, 321-326.

118. Goldberg, A.A.; Richard, V.R.; Kyryakov, P.; Bourque, S.D.; Beach, A.; Burstein, M.T.; Glebov, A.; Koupaki, O.; Boukh-Viner, T.; Gregg, C.; et al. Chemical genetic screen identifies lithocholic acid as an anti-aging compound that extends yeast chronological life span in a TOR-independent manner, by modulating housekeeping longevity assurance processes. Aging 2010, 2, 393-414.

119. Kaeberlein, M. Lessons on longevity from budding yeast. Nature 2010, 464, 513-519.

120. Kapahi, P; Chen, D.; Rogers, A.N.; Katewa, S.D.; Li, P.W.; Thomas, E.L.; Kockel, L. With TOR, less is more: A key role for the conserved nutrient-sensing TOR pathway in aging. Cell Metab. 2010, 11, 453-465.

121. Kenyon, C.J. The genetics of ageing. Nature 2010, 464, 504-512.

122. Longo, V.D.; Shadel, G.S.; Kaeberlein, M.; Kennedy, B. Replicative and chronological aging in Saccharomyces cerevisiae. Cell Metab. 2012, 16, 18-31.

123. Leonov, A.; Titorenko, V.I. A network of interorganellar communications underlies cellular aging. IUBMB Life 2013, 65, 665-674.

124. López-Otín, C.; Blasco, M.A.; Partridge, L.; Serrano, M.; Kroemer, G. The hallmarks of aging. Cell 2013, 153, 1194-1217.

125. Arlia-Ciommo, A.; Leonov, A.; Piano, A.; Svistkova, V.; Titorenko, V.I. Cell-autonomous mechanisms of chronological aging in the yeast Saccharomyces cerevisiae. Microbial Cell 2014, 1, 164-178.

126. Arlia-Ciommo, A.; Piano, A.; Leonov, A.; Svistkova, V.; Titorenko, V.I. Quasi-programmed aging of budding yeast: A trade-off between programmed processes of cell proliferation, differentiation, stress response, survival and death defines yeast lifespan. Cell Cycle 2014, 13, 3336-3349.

127. Guarente, L. Aging research-where do we stand and where are we going? Cell 2014, 159, 15-19.

128. Kennedy, B.K.; Berger, S.L.; Brunet, A.; Campisi, J.; Cuervo, A.M.; Epel, E.S.; Franceschi, C.; Lithgow, G.J.; Morimoto, R.I.; Pessin, J.E.; et al. Geroscience: Linking aging to chronic disease. Cell 2014, 159, 709-713.

129. Labbadia, J.; Morimoto, R.I. Proteostasis and longevity: When does aging really begin? F1000Prime Rep. 2014, 6, 7.

130. Partridge, L. Intervening in ageing to prevent the diseases of ageing. Trends Endocrinol. Metab. 2014, 25, 555-557.

131. Harel, I.; Benayoun, B.A.; Machado, B.; Singh, P.P.; Hu, C.K.; Pech, M.F.; Valenzano, D.R.; Zhang, E.; Sharp, S.C.; Artandi, S.E.; et al. A platform for rapid exploration of aging and diseases in a naturally short-lived vertebrate. Cell 2015, 160, 1013-1026.

132. Lee, D.; Hwang, W.; Artan, M.; Jeong, D.E.; Lee, S.J. Effects of nutritional components on aging. Aging Cell 2015, 14, 8-16.

133. Piano, A.; Titorenko, V.I. The intricate interplay between mechanisms underlying aging and cancer. Aging Dis. 2015, 6, 56-75.

134. Southam, C.M.; Ehrlich, J. Effects of extract of western red-cedar heartwood on certain wood-decaying fungi in culture. Phytopathology 1943, 33, 517-524.

135. Calabrese, E.J.; Baldwin, L.A. Defining hormesis. Hum. Exp. Toxicol. 2002, 21, 91-97. 
136. Mattson, M.P. Hormesis defined. Ageing Res. Rev. 2008, 7, 1-7.

137. Rattan, S.I. Hormesis in aging. Ageing Res. Rev. 2008, 7, 63-78.

138. Calabrese, E.J.; Bachmann, K.A.; Bailer, A.J.; Bolger, P.M.; Borak, J.; Cai, L.; Cedergreen, N.; Cherian, M.G.; Chiueh, C.C.; Clarkson, T.W.; et al. Biological stress response terminology: Integrating the concepts of adaptive response and preconditioning stress within a hormetic dose-response framework. Toxicol. Appl. Pharmacol. 2007, 222, 122-128.

139. Calabrese, E.J.; Mattson, M.P. Hormesis provides a generalized quantitative estimate of biological plasticity. J. Cell Commun. Signal. 2011, 5, 25-38.

140. Calabrese, E.J. Paradigm lost, paradigm found: The re-emergence of hormesis as a fundamental dose response model in the toxicological sciences. Environ. Pollut. 2005, 138, 379-411.

141. Lamming, D.W.; Wood, J.G.; Sinclair, D.A. Small molecules that regulate lifespan: Evidence for xenohormesis. Mol. Microbiol. 2004, 53, 1003-1009.

142. Howitz, K.T.; Sinclair, D.A. Xenohormesis: Sensing the chemical cues of other species. Cell 2008, $133,387-391$.

143. Dasgupta, B.; Milbrandt, J. Resveratrol stimulates AMP kinase activity in neurons. Proc. Natl. Acad. Sci. USA 2007, 104, 7217-7222.

144. Armour, S.M.; Baur, J.A.; Hsieh, S.N.; Land-Bracha, A.; Thomas, S.M.; Sinclair, D.A. Inhibition of mammalian S6 kinase by resveratrol suppresses autophagy. Aging 2009, 1, 515-528.

145. Blagosklonny, M.V. Inhibition of S6K by resveratrol: In search of the purpose. Aging 2009, 1, 511-514.

146. Demidenko, Z.N.; Blagosklonny, M.V. At concentrations that inhibit mTOR, resveratrol suppresses cellular senescence. Cell Cycle 2009, 8, 1901-1904.

147. Blagosklonny, M.V. Rapamycin and quasi-programmed aging: Four years later. Cell Cycle 2010, 9, 1859-1862.

148. Goldberg, A.A.; Kyryakov, P.; Bourque, S.D.; Titorenko, V.I. Xenohormetic, hormetic and cytostatic selective forces driving longevity at the ecosystemic level. Aging 2010, 2, 361-370.

149. Burstein, M.T.; Beach, A.; Richard, V.R.; Koupaki, O.; Gomez-Perez, A.; Goldberg, A.A.; Kyryakov, P.; Bourque, S.D.; Glebov, A.; Titorenko, V.I. Interspecies chemical signals released into the environment may create xenohormetic, hormetic and cytostatic selective forces that drive the ecosystemic evolution of longevity regulation mechanisms. Dose-Response 2012, 10, 75-82.

150. Vigh, L.; Horvàth, I.; van Hasselt, P.R.; Kuiper, P.J. Effect of frost hardening on lipid and fatty acid composition of chloroplast thylakoid membranes in two wheat varieties of contrasting hardiness. Plant Physiol. 1985, 79, 756-759.

151. Quinn, P.J.; Joo, F.; Vigh, L. The role of unsaturated lipids in membrane structure and stability. Prog. Biophys. Mol. Biol. 1989, 53, 71-103.

152. Balogh, G.; Horváth, I.; Nagy, E.; Hoyk, Z.; Benkõ, S.; Bensaude, O.; Vígh, L. The hyperfluidization of mammalian cell membranes acts as a signal to initiate the heat shock protein response. FEBS J. 2005, 272, 6077-6086.

153. Nagy, E.; Balogi, Z.; Gombos, I.; Akerfelt, M.; Björkbom, A.; Balogh, G.; Török, Z.; Maslyanko, A.; Fiszer-Kierzkowska, A.; Lisowska, K.; et al. Hyperfluidization-coupled membrane microdomain reorganization is linked to activation of the heat shock response in a murine melanoma cell line. Proc. Natl. Acad. Sci. USA 2007, 104, 7945-7950. 
154. Libertini, G. An adaptive theory of increasing mortality with increasing chronological age in populations in the wild. J. Theor. Biol. 1988, 132, 145-162.

155. Kirkwood, T.B. Comparative life spans of species: Why do species have the life spans they do? Am. J. Clin. Nutr. 1992, 55, 1191S-1195S.

156. Skulachev, V.P. Aging is a specific biological function rather than the result of a disorder in complex living systems: Biochemical evidence in support of Weismann's hypothesis. Biochemistry 1997, 62, 1191-1195.

157. Longo, V.D.; Mitteldorf, J.; Skulachev, V.P. Programmed and altruistic ageing. Nat. Rev. Genet. 2005, 6, 866-872.

158. Goldsmith, T.C. Aging, evolvability, and the individual benefit requirement; medical implications of aging theory controversies. J. Theor. Biol. 2008, 252, 764-768.

159. Ljubuncic, P.; Reznick, A.Z. The evolutionary theories of aging revisited-A mini-review. Gerontology 2009, 55, 205-216.

160. Mitteldorf, J. Aging is not a process of wear and tear. Rejuvenation Res. 2010, 13, 322-326.

161. Goldsmith, T.C. On the programmed/non-programmed aging controversy. Biochemistry 2012, 77, 729-732.

162. Mitteldorf, J. Adaptive aging in the context of evolutionary theory. Biochemistry 2012, 77, 716-725.

163. Trindade, L.S.; Aigaki, T.; Peixoto, A.A.; Balduino, A.; Mânica da Cruz, I.B.; Heddle, J.G. A novel classification system for evolutionary aging theories. Front. Genet. 2013, 4, 25.

(C) 2015 by the authors; licensee MDPI, Basel, Switzerland. This article is an open access article distributed under the terms and conditions of the Creative Commons Attribution license (http://creativecommons.org/licenses/by/4.0/). 